JOHnSON, EMILY NANCE, M.A. Permutation Tests for Mixed Paired and TwoSample Designs. (2018)

Directed by Dr. Scott Richter. 44 pp.

In this thesis we propose permutation tests of previously developed statistics for the case of mixed paired and two-sample data. We also explore the different weighting schemes of previous tests to understand the strengths and weaknesses of each test. We compare the power and Type I error of our new tests and those previously developed through a simulation. Rank based statistics generally performed as well as if not better than parametric statistics for all distributions. 


\title{
PERMUTATION TESTS FOR MIXED PAIRED AND TWO-SAMPLE DESIGNS
}

by

\author{
Emily Nance Johnson
}

\author{
A Thesis Submitted to \\ the Faculty of The Graduate School at \\ The University of North Carolina at Greensboro \\ in Partial Fulfillment \\ of the Requirements for the Degree \\ Master of Arts
}

Greensboro

2018

Approved by

Committee Chair 


\section{APPROVAL PAGE}

This thesis written by Emily Nance Johnson has been approved by the following committee of the Faculty of The Graduate School at The University of North Carolina at Greensboro.

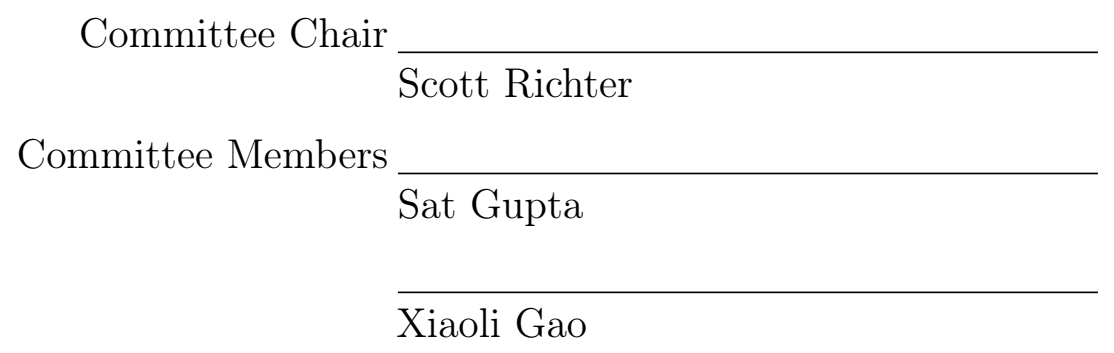

Date of Acceptance by Committee

Date of Final Oral Examination 


\section{TABLE OF CONTENTS}

Page

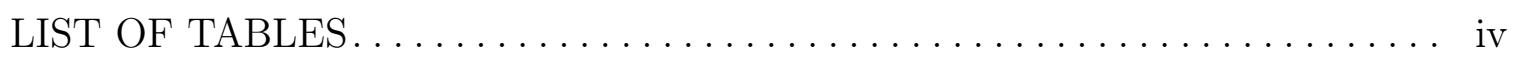
CHAPTER

I. INTRODUCTION $\ldots \ldots \ldots \ldots \ldots \ldots \ldots \ldots \ldots \ldots \ldots \ldots \ldots \ldots \ldots \ldots \ldots$

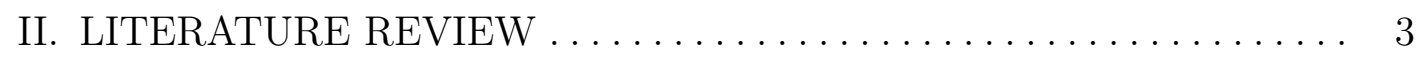

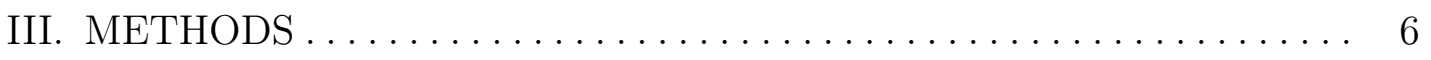

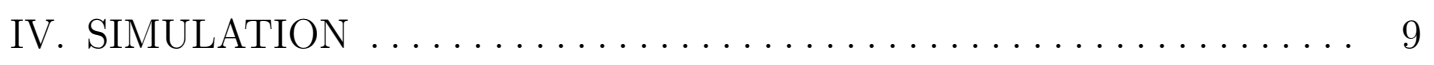

IV.1. Design $\ldots \ldots \ldots \ldots \ldots \ldots \ldots \ldots \ldots \ldots \ldots \ldots \ldots \ldots \ldots \ldots \ldots \ldots \ldots \ldots$

IV.2. Results . . . . . . . . . . . . . . . . . . 10

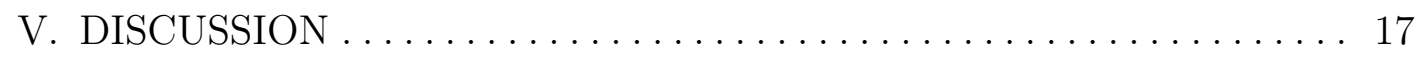

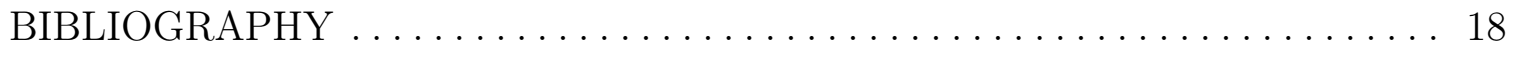

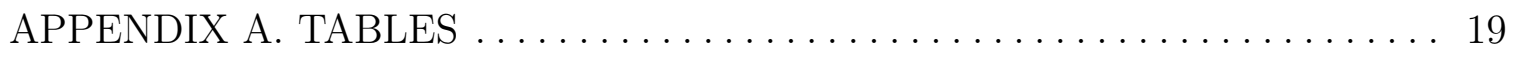

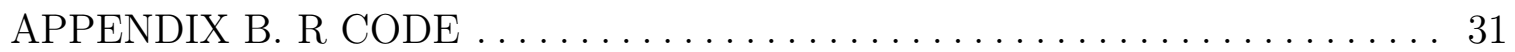




\section{LIST OF TABLES}

Table IV.1. Heavy-Tailed Skewed Distribution with $n_{p}=5, n_{u x}=7$, $n_{u y}=8 \ldots \ldots \ldots \ldots \ldots \ldots \ldots \ldots \ldots \ldots \ldots \ldots \ldots \ldots \ldots \ldots \ldots \ldots$

Table IV.2. Heavy-Tailed Skewed Distribution with $n_{p}=8, n_{u x}=16$, $n_{u y}=16 \ldots \ldots \ldots \ldots \ldots \ldots \ldots \ldots \ldots \ldots \ldots \ldots \ldots \ldots \ldots$

Table IV.3. Heavy-Tailed Symmetric Distribution with $n_{p}=32, n_{u x}=4$, $n_{u y}=4 \ldots \ldots \ldots \ldots \ldots \ldots \ldots \ldots \ldots \ldots \ldots \ldots \ldots \ldots \ldots \ldots \ldots$

Table IV.4. Heavy-Tailed Skewed Distribution with $n_{p}=10, n_{u x}=5$, $n_{u y}=5 \ldots \ldots \ldots \ldots \ldots \ldots \ldots \ldots \ldots \ldots \ldots \ldots \ldots \ldots \ldots \ldots \ldots \ldots$

Table IV.5. Heavy-Tailed Symmetric Distribution with $n_{p}=10, n_{u x}=5$, $n_{u y}=5 \ldots \ldots \ldots \ldots \ldots \ldots \ldots \ldots \ldots \ldots \ldots \ldots \ldots \ldots \ldots \ldots \ldots \ldots$

Table IV.6. Light-Tailed Skewed Distribution with $n_{p}=5, n_{u x}=7$,

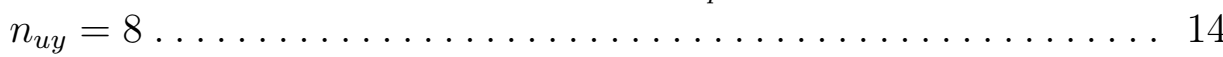

Table IV.7. Light-Tailed Skewed Distribution with $n_{p}=10, n_{u x}=5$, $n_{u y}=5 \ldots \ldots \ldots \ldots \ldots \ldots \ldots \ldots \ldots \ldots \ldots \ldots \ldots \ldots \ldots \ldots \ldots$

Table IV.8. Light-Tailed Skewed Distribution with $n_{p}=20, n_{u x}=10$,

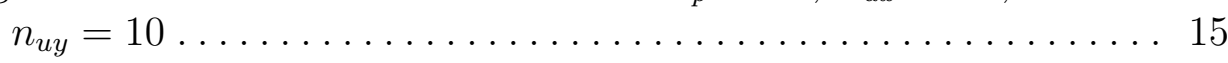

Table IV.9. Light-Tailed Skewed Distribution with $n_{p}=20, n_{u x}=30$, $n_{u y}=10 \ldots \ldots \ldots \ldots \ldots \ldots \ldots \ldots \ldots \ldots \ldots \ldots \ldots \ldots \ldots \ldots \ldots \ldots \ldots$

Table IV.10. Normal Distribution with $n_{p}=10, n_{u x}=5, n_{u y}=5 \ldots \ldots \ldots$

Table IV.11. Normal Distribution with $n_{p}=20, n_{u x}=10, n_{u y}=10 \ldots \ldots \ldots$

Table IV.12. Normal Distribution with $n_{p}=5, n_{u x}=7, n_{u y}=8 \ldots \ldots \ldots$

Table A.1. Normal Distribution with $n_{p}=10, n_{u x}=0, n_{u y}=0 \ldots \ldots \ldots \ldots$

Table A.2. Normal Distribution with $n_{p}=5, n_{u x}=2, n_{u y}=3 \ldots \ldots \ldots \ldots$

Table A.3. Normal Distribution with $n_{p}=20, n_{u x}=0, n_{u y}=0 \ldots \ldots \ldots \ldots$

Table A.4. Normal Distribution with $n_{p}=15, n_{u x}=2, n_{u y}=3 \ldots \ldots \ldots$

Table A.5. Normal Distribution with $n_{p}=40, n_{u x}=0, n_{u y}=0 \ldots \ldots \ldots \ldots$ 
Table A.6. Normal Distribution with $n_{p}=32, n_{u x}=4, n_{u y}=4 \ldots \ldots \ldots \ldots 21$

Table A.7. Normal Distribution with $n_{p}=8, n_{u x}=16, n_{u y}=16 \ldots \ldots \ldots 21$

Table A.8. Normal Distribution with $n_{p}=10, n_{u x}=5, n_{u y}=10 \ldots \ldots \ldots \ldots 21$

Table A.9. Normal Distribution with $n_{p}=20, n_{u x}=30, n_{u y}=10 \ldots \ldots \ldots 22$

Table A.10. Light-Tailed Skewed Distribution with $n_{p}=10, n_{u x}=0$, $n_{u y}=0 \ldots \ldots \ldots \ldots \ldots \ldots \ldots \ldots \ldots \ldots \ldots \ldots \ldots \ldots \ldots \ldots \ldots \ldots \ldots \ldots$

Table A.11. Light-Tailed Skewed Distribution with $n_{p}=5, n_{u x}=2$, $n_{u y}=3 \ldots \ldots \ldots \ldots \ldots \ldots \ldots \ldots \ldots \ldots \ldots \ldots \ldots \ldots \ldots \ldots \ldots \ldots \ldots$

Table A.12. Light-Tailed Skewed Distribution with $n_{p}=20, n_{u x}=0$,

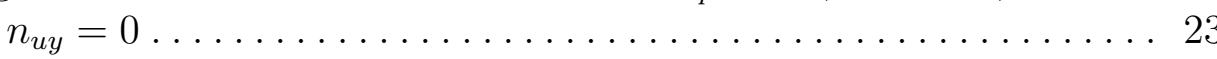

Table A.13. Light-Tailed Skewed Distribution with $n_{p}=15, n_{u x}=2$, $n_{u y}=3 \ldots \ldots \ldots \ldots \ldots \ldots \ldots \ldots \ldots \ldots \ldots \ldots \ldots \ldots \ldots \ldots \ldots$

Table A.14. Light-Tailed Skewed Distribution with $n_{p}=40, n_{u x}=0$,

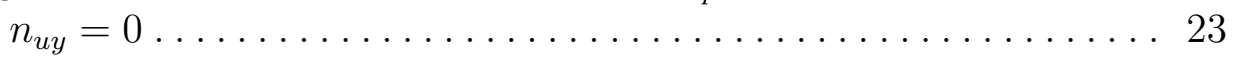

Table A.15. Light-Tailed Skewed Distribution with $n_{p}=32, n_{u x}=4$,

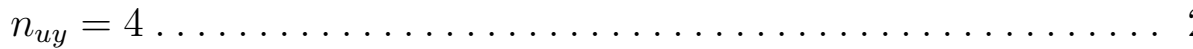

Table A.16. Light-Tailed Skewed Distribution with $n_{p}=8, n_{u x}=16$, $n_{u y}=16 \ldots \ldots \ldots \ldots \ldots \ldots \ldots \ldots \ldots \ldots \ldots \ldots \ldots \ldots \ldots \ldots \ldots \ldots \ldots$

Table A.17. Light-Tailed Skewed Distribution with $n_{p}=10, n_{u x}=5$, $n_{u y}=10 \ldots \ldots \ldots \ldots \ldots \ldots \ldots \ldots \ldots \ldots \ldots \ldots \ldots \ldots \ldots \ldots \ldots \ldots$

Table A.18. Heavy-Tailed Skewed Distribution with $n_{p}=10, n_{u x}=0$, $n_{u y}=0 \ldots \ldots \ldots \ldots \ldots \ldots \ldots \ldots \ldots \ldots \ldots \ldots \ldots \ldots \ldots \ldots \ldots \ldots$

Table A.19. Heavy-Tailed Skewed Distribution with $n_{p}=5, n_{u x}=2$,

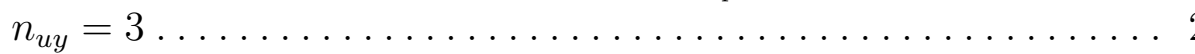

Table A.20. Heavy-Tailed Skewed Distribution with $n_{p}=20, n_{u x}=0$, $n_{u y}=0 \ldots \ldots \ldots \ldots \ldots \ldots \ldots \ldots \ldots \ldots \ldots \ldots \ldots \ldots \ldots \ldots \ldots \ldots \ldots$

Table A.21. Heavy-Tailed Skewed Distribution with $n_{p}=15, n_{u x}=2$, $n_{u y}=3 \ldots \ldots \ldots \ldots \ldots \ldots \ldots \ldots \ldots \ldots \ldots \ldots \ldots \ldots \ldots \ldots \ldots \ldots \ldots$

Table A.22. Heavy-Tailed Skewed Distribution with $n_{p}=40, n_{u x}=0$, $n_{u y}=0$ 
Table A.23. Heavy-Tailed Skewed Distribution with $n_{p}=32, n_{u x}=4$, $n_{u y}=4 \ldots \ldots \ldots \ldots \ldots \ldots \ldots \ldots \ldots \ldots \ldots \ldots \ldots \ldots \ldots \ldots \ldots \ldots \ldots$

Table A.24. Heavy-Tailed Skewed Distribution with $n_{p}=20, n_{u x}=10$, $n_{u y}=10 \ldots \ldots \ldots \ldots \ldots \ldots \ldots \ldots \ldots \ldots \ldots \ldots \ldots \ldots \ldots \ldots \ldots$

Table A.25. Heavy-Tailed Symmetric Distribution with $n_{p}=10, n_{u x}=0$, $n_{u y}=0 \ldots \ldots \ldots \ldots \ldots \ldots \ldots \ldots \ldots \ldots \ldots \ldots \ldots \ldots \ldots \ldots \ldots \ldots$

Table A.26. Heavy-Tailed Symmetric Distribution with $n_{p}=5, n_{u x}=2$, $n_{u y}=3$.

Table A.27. Heavy-Tailed Symmetric Distribution with $n_{p}=20, n_{u x}=0$, $n_{u y}=0$.

Table A.28. Heavy-Tailed Symmetric Distribution with $n_{p}=15, n_{u x}=2$,

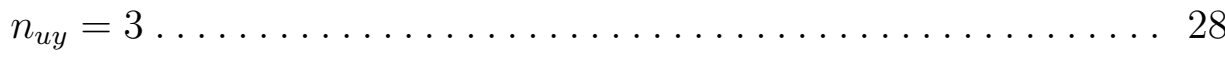

Table A.29. Heavy-Tailed Symmetric Distribution with $n_{p}=10, n_{u x}=5$,

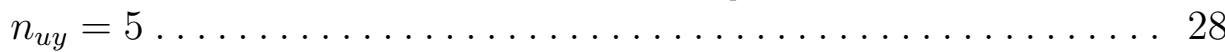

Table A.30. Heavy-Tailed Symmetric Distribution with $n_{p}=5, n_{u x}=7$, $n_{u y}=8 \ldots \ldots \ldots \ldots \ldots \ldots \ldots \ldots \ldots \ldots \ldots \ldots \ldots \ldots \ldots \ldots \ldots \ldots \ldots$

Table A.31. Heavy-Tailed Symmetric Distribution with $n_{p}=40, n_{u x}=0$,

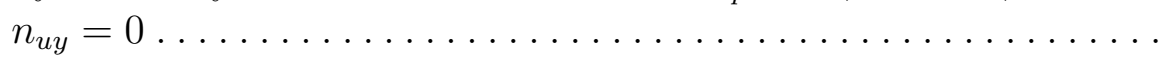

Table A.32. Heavy-Tailed Symmetric Distribution with $n_{p}=20, n_{u x}=10$, $n_{u y}=10$.

Table A.33. Heavy-Tailed Symmetric Distribution with $n_{p}=8, n_{u x}=16$,

$n_{u y}=16$ 


\section{CHAPTER I INTRODUCTION}

In certain experiments and observational studies for paired data, the issue of missing data may arise from several circumstances. Longitudinal studies will often involve missing observations at either time point. Other studies designed for two treatments to be applied to the same subject may also suffer from incomplete pairs if some subjects are not eligible for one treatment. This leads to a mixed experimental design with paired data and data that appear to be independent random samples from the two groups. Since the 1970's, various methods have been proposed in an effort to retain as much information as possible in the presence of some incomplete pairs. We are particularly interested in nonparametric tests and a way of combining two test statistics that would optimally account for both the complete and incomplete pairs. Dubnicka et al. [3] proposed both unweighted and weighted standardized sums of Wilcoxon signed-rank and Wilcoxon rank-sum statistics. Magel and Fu [7] proposed a similar test statistic to the unweighted version of Dubnicka et al., but standardized the Wilcoxon signed-rank and rank-sum statistics before adding them. This new statistic generally had higher power when there were more incomplete than complete pairs, especially under a normal distribution. Einsporn and Habtzghi [4] proposed a permutation test on the weighted mean difference that performed with higher power than the weighted test of Dubnicka et al. [3] for data generated from a normal distribution but not from an exponential distribution. Those were also the only two distributions considered by Einsporn and Habtzghi along with only two sets of sample sizes. 
We develop new permutation tests that combine the strengths of those previously proposed. We then conduct a simulation to investigate how these statistics perform relative to previously proposed methods for several combinations of distributions and sample sizes. 


\section{CHAPTER II LITERATURE REVIEW}

Numerous methods have been developed to analyze incomplete pairs, and many of the early approaches involved parametric tests. Lin and Stivers [6] proposed several adaptations to the paired and unpaired $t$-tests, and the choice of test statistic depended upon the circumstances of the study. One of those statistics was somewhat limited in that it was specifically designed for the case of equal variances. It was approximated by a $t$-distribution and was found to perform best when the paired sample size was between 5 and 20 .

Bhoj [1] proposed a weighted average of the paired and independent $t$ statistics for distributions with equal variances. This statistic allowed the researcher to adjust the relative weight of each statistic to maximize its sensitivity over the standard paired $t$-test and was found to be often more precise than the $t$-test when the data had high correlation and the sample sizes of the incomplete pairs were relatively small compared to the complete pairs. Bhoj [2] later developed better asymptotic approximations for the tests that further increased power beyond that of the $t$-test.

None of the statistics developed before the 1990's had known distributions but instead were based on approximate asymptotic distributions. Maritz [8] proposed an exact permutation test, but the incomplete pairs were permuted in a way that may not be considered standard. For all pairs, including those with a missing value, the observations were randomly interchanged within each pair. This assumed that each variable was equally likely to have missing values for the incomplete pairs and included the extreme cases where all missing values may be for one variable. Dubnicka et al. 
[3] proposed combining nonparametric statistics by using the Wilcoxon signed-rank statistic for complete pairs and Wilcoxon rank-sum statistic for incomplete pairs. They proposed both unweighted and weighted combinations of the statistics based on the asymptotic distributions of the nonparametric statistics. Magel and Fu [7] proposed a modification of the statistic of Dubnicka et al. [3] by standardizing the Wilcoxon signed-rank and Wilcoxon rank-sum statistics before adding them, whereas Dubnicka had suggested standardizing the sum. Magel and $\mathrm{Fu}$ found their test to be superior in some scenarios: it generally had higher power when there were more incomplete than complete pairs for all normal distributions as well as the exponential and $t$-distributions with unequal variances. However, when there were equal variances under the exponential distribution, the test usually had higher power when there were more complete pairs, and had higher power more consistently for the $t$-distributions with equal variances.

Einsporn and Habtzghi [4] proposed a permutation test that was a combination of standard permutation tests for means of the paired and independent samples. Unlike the permutation scheme proposed by Maritz, they chose to permute the incomplete pairs such that the sample size for each variable remained fixed in all arrangements. Their statistic was a weighted combination of the difference in means of the variables between the complete and incomplete pairs. They included a limited simulation that only considered two sets of sample sizes with normal and exponential underlying distributions. Their proposed test usually had marginally higher power than older tests proposed by Bhoj [2], Lin and Stivers [6], Maritz [8], and Dubnicka et al. [3] for small sample sizes and low correlation under a normal distribution. Bhoj's statistic [2] had the highest power of the parametric procedures considered and was often better than Einsporn and Habtzghi's [4] permutation test for high correlation and large 
sample sizes. The weighted nonparametric statistic proposed by Dubnicka et al. [3] almost always had highest power when the data were exponential. It was also apparent that the power of the Bhoj [2] test increased faster than that of the permutation test as the correlation increased and had higher power when the incomplete pairs' samples sizes became much larger than that of the complete pairs. Dubnicka's [3] weighted statistic also had higher power than the unweighted statistic as the correlation increased. Einsporn and Habtzghi's [4] statistic had power similar to or slightly higher than Dubnicka's [3] weighted statistic for data from a normal distribution. When the data came from an exponential distribution, Dubnicka's [3] weighted statistic always performed better. This suggests that using the combination of Wilcoxon signed-rank and Wilcoxon rank-sum statistics may be a better choice in most practical situations since the true distribution will not be known.

The nonparametric tests generally performed better in the limited simulations of Einsporn and Habtzghi [4], but a wider variety of conditions, such as heavier-tailed distributions and different sample size combinations, may need to be considered to better compare the different methods. Einsporn and Habtzghi [4] found that the permutation test on means tended to have higher power than Bhoj's parametric asymptotic test on means [2]. This suggests that a permutation version of the rank statistics may also perform better than the asymptotic versions, especially for small to moderate sample sizes. Thus, we propose permutation versions of the rank statistics developed by Dubnicka et al. [3] and compare the new permutation methods to previously considered statistics over a wide range of conditions. 


\section{CHAPTER III}

\section{METHODS}

We compared the two new permutation rank statistics to that of Einsporn and Habtzghi [4], Magel and Fu [7], Bhoj [2], and both weighted and unweighted statistics of Dubnicka et al. [3]. These were chosen to examine both parametric and nonparametric tests that had been competitive in previous simulations. The standard paired $t$-test and Wilcoxon signed-rank tests were also included to compare to the case of discarding the incomplete pairs.

Bhoj [1] proposed a weighted sum of paired and independent $t$ tests of the form $Z=\frac{w T_{1}+(1-w) T_{2}}{D}$ where $T_{1}$ and $T_{2}$ were the usual independent and paired $t$-statistics respectively and $D$ a multiplier to help achieve an approximate $t$-distribution. They derived two complex transformations to achieve a better approximation, which resulted in $Z_{b}=\frac{\lambda_{b} U_{1}+\left(1-\lambda_{b}\right) U_{3}}{\sqrt{\lambda_{b}^{2}+\left(1-\lambda_{b}\right)^{2}}}$ where $U_{1}$ and $U_{3}$ were the transformed $t$-statistics and $\lambda_{b}$ the weight for the transformed statistics. See Bhoj [2] for exact transformation details. In a simulation comparing several statistics, including that of Lin and Stivers [6], Bhoj's $Z_{b}$ statistic had higher power than that of Lin and Stivers for small sample sizes and high correlation and in general for larger sample sizes.

Dubnicka et al. [3] developed nonparametric tests that were weighted sums of Wilcoxon signed-rank and rank-sum statistics. The Wilcoxon signed-rank statistic is calculated by computing the difference within each pair and ranking the differences without regard to their sign. The signs are then reattached to the ranks, and the positive and negative ranks are added separately. Then the test statistic $S$ is the sum of the positive ranks. The rank-sum statistic is calculated by combining the 
values from both samples and then assigning ranks to the combined data. The ranks for each sample are added, and $U$ is the smaller of the two sums. The signed-rank statistic has mean $E(S)=\frac{n(n+1)}{4}$ and variance $\operatorname{Var}(S)=\frac{n(n+1)(2 n+1)}{24}$, and the ranksum statistic has mean $E(U)=\frac{n_{1} n_{2}}{2}$ and variance $\operatorname{Var}(U)=\frac{n_{1} n_{2}\left(n_{1}+n_{2}+1\right)}{12}$. The unweighted statistic suggested by Dubnicka [3] was by $R=S+U$, which has mean $E(R)=\frac{n(n+1)}{4}+\frac{n_{1} n_{2}}{2}$ and variance $\operatorname{Var}(R)=\frac{n(n+1)(2 n+1)}{24}+\frac{n_{1} n_{2}\left(n_{1}+n_{2}+1\right)}{12}$. Then the statistic $R_{z}=\frac{R-E(R)}{\sqrt{\operatorname{Var}(R)}}$ is assumed to follow an approximate $\mathcal{N}(0,1)$ distribution. They also considered a weighted statistic, $R_{w}=\frac{2\left(n_{1}+n_{2}\right)}{\left(n\left(n_{1}+n_{2}\right)+2 n_{1} n_{2}\right)(n+1)} S+\frac{2}{n\left(n_{1}+n_{2}\right)+2 n_{1} n_{2}} U$, with mean $E\left(R_{w}\right)=\frac{1}{2}$ and variance equal to the weighted sum of the variances of the Wilcoxon signed-rank and rank-sum statistics. The statistic $R_{w, z}=\frac{R_{w}-\frac{1}{2}}{\sqrt{\operatorname{Var}\left(R_{w}\right)}}$ is also assumed to follow an approximate $\mathcal{N}(0,1)$ distribution. The weights were derived to be optimal under a bivariate normal distribution with $\rho=0.5$, and Dubnicka et al. [3] found that the weighted rank statistic was generally more efficient than the unweighted statistic. If the number of complete pairs was more than the sum of the incomplete pairs, though, the unweighted statistic was nearly as efficient. Thus, they recommended the simpler unweighted statistic in those cases. Magel and Fu [9] proposed a slight variation of Dubnicka et al. [3] in which they standardized the Wilcoxon signed-rank and rank-sum statistics before combining them. The resulting statistic was $M=\frac{S^{*}+U^{*}}{\sqrt{2}}$, where $S^{*}$ and $U^{*}$ were standardized Wilcoxon signedrank and rank-sum statistics respectively. They included a simulation in which they compared the asymptotic unweighted statistic $R_{z}$ of Dubnicka et al. [3] to their own with varying samples sizes for normal, exponential, and $t$ distributions. The power of $M$ was generally only higher with more incomplete pairs than complete pairs.

Einsporn and Habtzghi [4] proposed a permutation test based on weighted mean differences similar to the asymptotic test from Bhoj [2] and defined it as 
$T=w \bar{d}_{p}+(1-w) \bar{d}_{u}$ with weight $w=\left(\frac{1}{n_{u x}}+\frac{1}{n_{u y}}\right) /\left(\frac{2-2 \rho}{n_{p}}+\frac{1}{n_{u x}}+\frac{1}{n_{u y}}\right)$ that minimized the variance of $T$. This statistic generally performed better for low correlation and small sample sizes under the normal distribution. Specifically, it had roughly the same or higher power than Bhoj's $Z_{b}[2]$ statistic in those cases. However, the asymptotic nonparametric tests from Dubnicka et al. [3] tended to have the highest power for the exponential distributions. From these observations we expect that a permutation version of the nonparametric tests may have higher power than the asymptotic versions for such cases and also $T$ for nonnormal distributions.

The new methods we propose are permutation versions of the rank tests of Dubnicka [3]. We permute the data following the method of Einsporn and Habtzghi [4]. For $n_{p}$ complete pairs, generate $n_{p}$ uniform $(0,1)$ random variables. The observations in a pair are reversed if the uniform random variable is greater than 0.5. For the incomplete pairs the observations are combined into one vector where the first $n_{u x}$ values are the observed $x$ 's, and the remaining $n_{u y}$ values are the observed $y$ 's. For each permutation, a random selection of size $n_{u x}$ are chosen to be $x$ 's and the remaining observations become $y$ 's. Both the unweighted $R_{\text {perm }}=S+U$ and weighted $R_{w, p e r m}=\frac{2\left(n_{1}+n_{2}\right)}{\left(n\left(n_{1}+n_{2}\right)+2 n_{1} n_{2}\right)(n+1)} S+\frac{2}{n\left(n_{1}+n_{2}\right)+2 n_{1} n_{2}} U$ statistics are calculated on the observed values and for each permutation. Standardizing the statistic is not necessary because the mean and variance are based only on sample sizes and thus remain constant for all permutations. The one-sided $p$-values are then the proportion of permutations with statistics greater than or equal to the observed statistic. 


\section{CHAPTER IV}

\section{SIMULATION}

\section{IV.1. Design}

We examined the power and Type I error properties of the tests described in Chapter III through a simulation. Four different distributions were assumed to generate the data: (i) standard normal, (ii) skewed light-tailed, (iii) skewed heavytailed, and (iv) symmetric heavy-tailed. We used the $g$ and $h$ distributions [5] to generate the data. For these distributions, the $g$ value controls the skewness of the distribution, and the $h$ value controls the elongation of the distribution tails [5]. We used $g=0.7$ and $h=0$ for the skewed light-tailed distributions which will have skewness and tail weight similar to an exponential distribution, $g=0.7$ and $h=0.35$ for the skewed heavy-tailed distributions, and $g=0$ and $h=0.4$ for the symmetric heavy-tailed distributions. Since the data were assumed to be generated as matchedpairs with possible incomplete pairs, we assumed that the paired samples had equal variance $\sigma^{2}=1$ and Pearson correlation values of $\rho=0.1,0.5$, and 0.9 . In order to generate correlated variables under all distributions we first generated random data from a bivariate normal distribution with means $\mu_{X}$ and $\mu_{Y}$ and then used their probabilities to generate data under the new distribution in such a way that the Spearman correlation between the normally distributed variables and transformed variables were equal. Once correlated pairs were generated, observations were randomly deleted from each variable separately to simulate incomplete pairs. We obtained the sample Spearman correlation from the remaining complete pairs for calculating the weights of certain test statistics. We estimated Type I error with a true difference 
of $\delta=\mu_{X}-\mu_{Y}=0$ and estimated power for $\delta=0.5$ and 1. For a few cases we added $\delta=0.25$ to better distinguish between the tests when the power reached 1 very quickly.

We included total sample sizes of 10,20 , and 40 with small, moderate, and large percentages of missing data. The small sample size only allowed us to consider $50 \%$ missing, while we included 25\%,50\%, and $75 \%$ missing for the moderate sample size and 20\%,50\%, and $80 \%$ missing for the large sample size. We also included the two sets of sample sizes presented by Einsporn and Habtzghi [4] for comparison. For each distribution, mean difference, sample size, and correlation, Type I error or power was estimated as the proportion of rejections out of 5000 data sets. For each permutation test 566 random permutations were used to estimate the p-value. Marozzi [9] has shown that power only increases slightly with more permutations, and the maximum root mean squared error of the estimation is only 0.00849 rather than 0.005 if all permutations were considered. If estimates for two tests have a difference greater than at least $2 \times 0.00849=0.0168$, then we can be approximately $95 \%$ confident that one test result does in fact differ statistically from the other.

\section{IV.2. Results}

Type 1 error and power estimates are given in the tables below. When the data consisted of entirely complete pairs, the results for certain tests would be expected to be the same. The weighted and unweighted permutation statistics $R_{\text {perm }}$ and $R_{w, p e r m}$ will always have the same estimates for these cases because the signed rank statistic receives weight equal to 1 when there are no incomplete pairs. The $Z_{b}$ and $t$ statistics likewise will always have the same estimates for these cases because $Z_{b}$ reverts to a paired t-test for the same scenario, and $R_{z}, R_{w, z}, M$, and $S$ will always have the same values as they become the asymptotic Wilcoxon signed-rank test. In these cases the 
$T$ generally had the highest power for the normal distributions, but for moderate to high correlation and a sample size of 10, the $t$ test had the highest power (Tables A.1, A.3, and A.5). The asymptotic rank statistics had the highest power for all nonnormal distributions. The new permutation rank statistics $R_{\text {perm }}$ and $R_{w, p e r m}$ generally had higher power than the $T$ permutation statistic based on means for nonnormal distributions (Tables A.10, A.12, A.14, A.18, A.20, A.22, A.25, A.27, and A.31).

For both heavy-tailed skewed and symmetric distributions the rank based statistics generally had the highest power. In Table IV.1 with $75 \%$ incomplete pairs, $R_{z}$ had the highest power for low correlation while $M$ generally had the highest power for moderate to high correlation. For one case with $\delta=0.5$ and $\rho=0.9$, though, $S$ had the highest power even ignoring the complete pairs. With $80 \%$ incomplete pairs in Table IV.2, $R_{w, z}$ and $R_{w, p e r m}$ had the highest power for low and moderate correlation, but $M$ had the highest power with high correlation. The power of both $R_{w, z}$ and $R_{w, p e r m}$ grew faster than $R_{z}$ and $R_{\text {perm }}$ as the correlation increased. For the heavy-tailed symmetric distribution with only $20 \%$ incomplete pairs in Table IV.3, $R_{w, p e r m}$ generally had the highest power for low and moderate correlation, while $S$ was best when correlation was high. For moderate and large sample sizes with 50\% missing for both the skewed and symmetric distributions, $R_{z}$ had the highest power (Tables IV.4 and IV.5).

The rank-based statistics were also most powerful for the light-tailed skewed distribution. With $75 \%$ missing and a moderate sample size of $20, M$ generally had the highest power, as seen in Table IV.6. Table IV.7 shows another case with the same sample size but with $50 \%$ incomplete pairs where $R_{z}$ had the highest power for all combinations of correlation and mean differences. When the sample size doubled 
to 40, though, Table IV.8 shows that $R_{w, p e r m}$ had the highest power for $\delta=0.5$ and $\rho=0.1$ and $0.5, M$ had the highest power for $\delta=1$ and $\rho=0.1$, while $R_{w, z}$ had the highest power for the other cases. $R_{w, p e r m}$ also had the highest power for the unequal incomplete sample sizes Einsporn and Habtzghi [4] previously presented (Table IV.9).

For sample sizes of 20 and 40 with $50 \%$ incomplete pairs from a normal distribution in Tables IV.10 and IV.11, T had the highest power in all but one case. With $75 \%$ missing in Table IV.12, $T$ also had the highest power for low to moderate correlation, while $S$ achieved the highest power with high correlation.

For all of the cases with 10 or fewer incomplete pairs on each variable, the estimated Type I error rates of $Z_{b}$ were inflated and generally increased with correlation. This may be due to a poor asymptotic approximation for these cases, and further investigation into this statistic is needed to understand and address this issue.

Table IV.1. Heavy-Tailed Skewed Distribution with $n_{p}=5, n_{u x}=7, n_{u y}=8$. Empirical Size and Power at $\alpha=0.05$

\begin{tabular}{ccccccccccc}
\hline$\delta$ & $\rho$ & $T$ & $R_{z}$ & $R_{\text {perm }}$ & $R_{w, z}$ & $R_{w, \text { perm }}$ & $Z_{b}$ & $M$ & $t$ & $S$ \\
\hline 0 & 0.1 & 0.0598 & 0.0588 & 0.0494 & 0.0506 & 0.0518 & 0.0254 & 0.053 & 0.0326 & 0.0596 \\
0 & 0.5 & 0.063 & 0.058 & 0.049 & 0.0512 & 0.0528 & 0.0364 & 0.0536 & 0.0312 & 0.0588 \\
0 & 0.9 & 0.0576 & 0.0606 & 0.0518 & 0.05 & 0.0526 & 0.0524 & 0.0542 & 0.0318 & 0.0576 \\
0.5 & 0.1 & 0.195 & 0.25 & 0.2206 & 0.233 & 0.2326 & 0.0392 & 0.2412 & 0.0944 & 0.1632 \\
0.5 & 0.5 & 0.224 & 0.2774 & 0.2468 & 0.2698 & 0.2712 & 0.0682 & 0.2786 & 0.1264 & 0.209 \\
0.5 & 0.9 & 0.3762 & 0.3588 & 0.3188 & 0.429 & 0.4294 & 0.2822 & 0.4322 & 0.3438 & 0.4554 \\
1.0 & 0.1 & 0.3896 & 0.5524 & 0.5092 & 0.5278 & 0.5262 & 0.0798 & 0.538 & 0.2152 & 0.3136 \\
1.0 & 0.5 & 0.4406 & 0.5886 & 0.5462 & 0.6032 & 0.5998 & 0.16 & 0.6118 & 0.306 & 0.4112 \\
1.0 & 0.9 & 0.6478 & 0.679 & 0.6354 & 0.7682 & 0.7668 & 0.5786 & 0.7716 & 0.6524 & 0.7322 \\
\hline
\end{tabular}


Table IV.2. Heavy-Tailed Skewed Distribution with $n_{p}=8, n_{u x}=16, n_{u y}=16$. Empirical Size and Power at $\alpha=0.05$

\begin{tabular}{ccccccccccc}
\hline$\delta$ & $\rho$ & $T$ & $R_{z}$ & $R_{\text {perm }}$ & $R_{w, z}$ & $R_{w, \text { perm }}$ & $Z_{b}$ & $M$ & $t$ & $S$ \\
\hline 0 & 0.1 & 0.0584 & 0.0506 & 0.051 & 0.053 & 0.0524 & 0.0108 & 0.0528 & 0.032 & 0.051 \\
0 & 0.5 & 0.0594 & 0.0534 & 0.0532 & 0.052 & 0.053 & 0.0188 & 0.051 & 0.0296 & 0.0512 \\
0 & 0.9 & 0.056 & 0.049 & 0.0496 & 0.0508 & 0.0512 & 0.0304 & 0.0512 & 0.0304 & 0.0512 \\
0.5 & 0.1 & 0.2246 & 0.3572 & 0.355 & 0.3748 & 0.3706 & 0.0228 & 0.3724 & 0.1238 & 0.178 \\
0.5 & 0.5 & 0.2546 & 0.3732 & 0.3744 & 0.4264 & 0.4274 & 0.056 & 0.4244 & 0.166 & 0.2334 \\
0.5 & 0.9 & 0.4324 & 0.4492 & 0.4436 & 0.6328 & 0.631 & 0.3366 & 0.6368 & 0.4336 & 0.512 \\
1.0 & 0.1 & 0.4672 & 0.7762 & 0.776 & 0.7904 & 0.7908 & 0.068 & 0.7868 & 0.2866 & 0.3578 \\
1.0 & 0.5 & 0.5144 & 0.7984 & 0.796 & 0.849 & 0.8486 & 0.1674 & 0.8464 & 0.3854 & 0.4638 \\
1.0 & 0.9 & 0.7242 & 0.8482 & 0.8438 & 0.9508 & 0.949 & 0.6476 & 0.952 & 0.7248 & 0.7572 \\
\hline
\end{tabular}

Table IV.3. Heavy-Tailed Symmetric Distribution with $n_{p}=32, n_{u x}=4, n_{u y}=4$. Empirical Size and Power at $\alpha=0.05$

\begin{tabular}{ccccccccccc}
\hline$\delta$ & $\rho$ & $T$ & $R_{z}$ & $R_{\text {perm }}$ & $R_{w, z}$ & $R_{w, \text { perm }}$ & $Z_{b}$ & $M$ & $t$ & $S$ \\
\hline 0 & 0.1 & 0.052 & 0.0504 & 0.0496 & 0.0498 & 0.0524 & 0.08 & 0.0512 & 0.0456 & 0.0534 \\
0 & 0.5 & 0.0548 & 0.0514 & 0.0514 & 0.0472 & 0.0498 & 0.0834 & 0.0502 & 0.0454 & 0.0536 \\
0 & 0.9 & 0.0524 & 0.0504 & 0.0532 & 0.0506 & 0.053 & 0.0852 & 0.05 & 0.0416 & 0.0526 \\
0.5 & 0.1 & 0.309 & 0.3992 & 0.399 & 0.4224 & 0.4258 & 0.2716 & 0.3724 & 0.2798 & 0.3912 \\
0.5 & 0.5 & 0.4042 & 0.539 & 0.5368 & 0.5618 & 0.5586 & 0.3768 & 0.4762 & 0.377 & 0.533 \\
0.5 & 0.9 & 0.7858 & 0.9698 & 0.9694 & 0.9692 & 0.9692 & 0.7686 & 0.8476 & 0.7672 & 0.9704 \\
1.0 & 0.1 & 0.6502 & 0.8312 & 0.8296 & 0.8544 & 0.857 & 0.5994 & 0.7964 & 0.6082 & 0.82 \\
1.0 & 0.5 & 0.7742 & 0.9506 & 0.9492 & 0.9594 & 0.96 & 0.7408 & 0.8924 & 0.7434 & 0.944 \\
1.0 & 0.9 & 0.949 & 1 & 1 & 0.9998 & 0.9998 & 0.9398 & 0.9954 & 0.9398 & 1 \\
\hline
\end{tabular}

Table IV.4. Heavy-Tailed Skewed Distribution with $n_{p}=10, n_{u x}=5, n_{u y}=5$. Empirical Size and Power at $\alpha=0.05$

\begin{tabular}{ccccccccccc}
\hline$\delta$ & $\rho$ & $T$ & $R_{z}$ & $R_{\text {perm }}$ & $R_{w, z}$ & $R_{w, \text { perm }}$ & $Z_{b}$ & $M$ & $t$ & $S$ \\
\hline 0 & 0.1 & 0.0556 & 0.0546 & 0.0492 & 0.0516 & 0.051 & 0.0458 & 0.0504 & 0.0368 & 0.0506 \\
0 & 0.5 & 0.056 & 0.0546 & 0.0466 & 0.051 & 0.051 & 0.0542 & 0.0512 & 0.0344 & 0.0518 \\
0 & 0.9 & 0.0544 & 0.0546 & 0.0452 & 0.0494 & 0.0498 & 0.0708 & 0.0522 & 0.0334 & 0.05 \\
0.5 & 0.1 & 0.1974 & 0.2566 & 0.2312 & 0.245 & 0.2448 & 0.101 & 0.252 & 0.14 & 0.1968 \\
0.5 & 0.5 & 0.235 & 0.3296 & 0.292 & 0.3142 & 0.3102 & 0.1622 & 0.303 & 0.1854 & 0.256 \\
0.5 & 0.9 & 0.4872 & 0.6402 & 0.6034 & 0.6166 & 0.6142 & 0.4562 & 0.522 & 0.4644 & 0.6122 \\
1.0 & 0.1 & 0.3982 & 0.5678 & 0.5308 & 0.555 & 0.5548 & 0.2406 & 0.5618 & 0.3058 & 0.416 \\
1.0 & 0.5 & 0.4896 & 0.691 & 0.6566 & 0.6766 & 0.6762 & 0.3694 & 0.657 & 0.4244 & 0.557 \\
1.0 & 0.9 & 0.7572 & 0.9156 & 0.8974 & 0.9022 & 0.902 & 0.7354 & 0.8424 & 0.7428 & 0.893 \\
\hline
\end{tabular}


Table IV.5. Heavy-Tailed Symmetric Distribution with $n_{p}=10, n_{u x}=5, n_{u y}=5$. Empirical Size and Power at $\alpha=0.05$

\begin{tabular}{ccccccccccc}
\hline$\delta$ & $\rho$ & $T$ & $R_{z}$ & $R_{\text {perm }}$ & $R_{w, z}$ & $R_{w, \text { perm }}$ & $Z_{b}$ & $M$ & $t$ & $S$ \\
\hline 0 & 0.1 & 0.056 & 0.0564 & 0.0476 & 0.0528 & 0.0538 & 0.0604 & 0.0502 & 0.0416 & 0.0542 \\
0 & 0.5 & 0.0542 & 0.0562 & 0.0474 & 0.0512 & 0.0532 & 0.069 & 0.05 & 0.0416 & 0.0542 \\
0 & 0.9 & 0.0512 & 0.0542 & 0.0466 & 0.0514 & 0.0522 & 0.085 & 0.05 & 0.0398 & 0.0534 \\
0.5 & 0.1 & 0.2042 & 0.2502 & 0.2206 & 0.2386 & 0.2366 & 0.114 & 0.237 & 0.1498 & 0.1868 \\
0.5 & 0.5 & 0.2482 & 0.3108 & 0.2784 & 0.2958 & 0.2924 & 0.1714 & 0.273 & 0.1988 & 0.247 \\
0.5 & 0.9 & 0.5236 & 0.6226 & 0.5842 & 0.5976 & 0.596 & 0.5022 & 0.5032 & 0.5068 & 0.5962 \\
1.0 & 0.1 & 0.4492 & 0.5636 & 0.5188 & 0.5482 & 0.5412 & 0.272 & 0.5478 & 0.3448 & 0.4142 \\
1.0 & 0.5 & 0.5344 & 0.6716 & 0.639 & 0.6594 & 0.6582 & 0.4134 & 0.636 & 0.465 & 0.541 \\
1.0 & 0.9 & 0.8448 & 0.9348 & 0.9194 & 0.9246 & 0.9236 & 0.829 & 0.8522 & 0.8342 & 0.9188 \\
\hline
\end{tabular}

Table IV.6. Light-Tailed Skewed Distribution with $n_{p}=5, n_{u x}=7, n_{u y}=8$. Empirical Size and Power at $\alpha=0.05$

\begin{tabular}{ccccccccccc}
\hline$\delta$ & $\rho$ & $T$ & $R_{z}$ & $R_{\text {perm }}$ & $R_{w, z}$ & $R_{w, \text { perm }}$ & $Z_{b}$ & $M$ & $t$ & $S$ \\
\hline 0 & 0.1 & 0.061 & 0.0588 & 0.0502 & 0.048 & 0.0484 & 0.0292 & 0.0508 & 0.0366 & 0.0604 \\
0 & 0.5 & 0.0634 & 0.0576 & 0.0478 & 0.0512 & 0.0516 & 0.0438 & 0.054 & 0.0352 & 0.0606 \\
0 & 0.9 & 0.0608 & 0.06 & 0.0516 & 0.0516 & 0.0536 & 0.0638 & 0.0546 & 0.0342 & 0.0592 \\
0.5 & 0.1 & 0.285 & 0.3412 & 0.3012 & 0.3264 & 0.3268 & 0.0596 & 0.3356 & 0.1482 & 0.2112 \\
0.5 & 0.5 & 0.3346 & 0.3766 & 0.3378 & 0.3868 & 0.387 & 0.1192 & 0.3972 & 0.2132 & 0.2888 \\
0.5 & 0.9 & 0.557 & 0.4758 & 0.4292 & 0.5888 & 0.589 & 0.4668 & 0.5932 & 0.5372 & 0.6118 \\
1.0 & 0.1 & 0.6132 & 0.7252 & 0.6814 & 0.7152 & 0.7124 & 0.135 & 0.7258 & 0.342 & 0.4204 \\
1.0 & 0.5 & 0.6826 & 0.7672 & 0.7288 & 0.7862 & 0.785 & 0.2818 & 0.7934 & 0.4782 & 0.555 \\
1.0 & 0.9 & 0.8762 & 0.8276 & 0.8002 & 0.9106 & 0.9088 & 0.8018 & 0.9118 & 0.8638 & 0.8916 \\
\hline
\end{tabular}

Table IV.7. Light-Tailed Skewed Distribution with $n_{p}=10, n_{u x}=5, n_{u y}=5$. Empirical Size and Power at $\alpha=0.05$

\begin{tabular}{ccccccccccc}
\hline$\delta$ & $\rho$ & $T$ & $R_{z}$ & $R_{\text {perm }}$ & $R_{w, z}$ & $R_{w, \text { perm }}$ & $Z_{b}$ & $M$ & $t$ & $S$ \\
\hline 0 & 0.1 & 0.054 & 0.054 & 0.0466 & 0.0498 & 0.05 & 0.0624 & 0.0522 & 0.0448 & 0.0526 \\
0 & 0.5 & 0.0548 & 0.0548 & 0.0444 & 0.0512 & 0.0494 & 0.0728 & 0.0506 & 0.043 & 0.0526 \\
0 & 0.9 & 0.0524 & 0.0554 & 0.0446 & 0.0498 & 0.0502 & 0.0856 & 0.0512 & 0.0408 & 0.0528 \\
0.5 & 0.1 & 0.2988 & 0.3694 & 0.3312 & 0.3538 & 0.3564 & 0.1756 & 0.3578 & 0.2306 & 0.2718 \\
0.5 & 0.5 & 0.3816 & 0.4792 & 0.4384 & 0.463 & 0.4576 & 0.2846 & 0.438 & 0.3222 & 0.3864 \\
0.5 & 0.9 & 0.747 & 0.8232 & 0.7968 & 0.8034 & 0.8026 & 0.7306 & 0.6988 & 0.7352 & 0.808 \\
1.0 & 0.1 & 0.667 & 0.766 & 0.7382 & 0.757 & 0.7548 & 0.4372 & 0.756 & 0.531 & 0.595 \\
1.0 & 0.5 & 0.7626 & 0.871 & 0.8472 & 0.8612 & 0.8572 & 0.6418 & 0.837 & 0.688 & 0.7558 \\
1.0 & 0.9 & 0.9662 & 0.986 & 0.9818 & 0.9822 & 0.9822 & 0.9654 & 0.9492 & 0.9662 & 0.9842 \\
\hline
\end{tabular}


Table IV.8. Light-Tailed Skewed Distribution with $n_{p}=20, n_{u x}=10, n_{u y}=10$. Empirical Size and Power at $\alpha=0.05$

\begin{tabular}{ccccccccccc}
\hline$\delta$ & $\rho$ & $T$ & $R_{z}$ & $R_{\text {perm }}$ & $R_{w, z}$ & $R_{w, \text { perm }}$ & $Z_{b}$ & $M$ & $t$ & $S$ \\
\hline 0 & 0.1 & 0.0524 & 0.049 & 0.049 & 0.05 & 0.0508 & 0.0512 & 0.0538 & 0.0452 & 0.0472 \\
0 & 0.5 & 0.0524 & 0.0488 & 0.0502 & 0.0496 & 0.0516 & 0.0638 & 0.0532 & 0.046 & 0.0466 \\
0 & 0.9 & 0.052 & 0.0496 & 0.0516 & 0.0508 & 0.0532 & 0.0878 & 0.0526 & 0.046 & 0.047 \\
0.5 & 0.1 & 0.4408 & 0.5628 & 0.5634 & 0.5682 & 0.5726 & 0.2544 & 0.5718 & 0.3566 & 0.4146 \\
0.5 & 0.5 & 0.5548 & 0.7072 & 0.7042 & 0.7118 & 0.7122 & 0.4234 & 0.6894 & 0.4848 & 0.5958 \\
0.5 & 0.9 & 0.914 & 0.9796 & 0.9792 & 0.9798 & 0.979 & 0.8992 & 0.94 & 0.9032 & 0.9696 \\
1.0 & 0.1 & 0.8546 & 0.9542 & 0.9532 & 0.956 & 0.956 & 0.6516 & 0.9576 & 0.7458 & 0.8422 \\
1.0 & 0.5 & 0.9286 & 0.9888 & 0.9872 & 0.989 & 0.988 & 0.8428 & 0.9844 & 0.881 & 0.9516 \\
1.0 & 0.9 & 0.9978 & 1 & 1 & 1 & 1 & 0.9958 & 0.9992 & 0.996 & 1 \\
\hline
\end{tabular}

Table IV.9. Light-Tailed Skewed Distribution with $n_{p}=20, n_{u x}=30, n_{u y}=10$. Empirical Size and Power at $\alpha=0.05$

\begin{tabular}{ccccccccccc}
\hline$\delta$ & $\rho$ & $T$ & $R_{z}$ & $R_{\text {perm }}$ & $R_{w, z}$ & $R_{w, \text { perm }}$ & $Z_{b}$ & $M$ & $t$ & $S$ \\
\hline 0 & 0.1 & 0.0524 & 0.05 & 0.0496 & 0.0522 & 0.0524 & 0.0276 & 0.0492 & 0.0474 & 0.0506 \\
0 & 0.5 & 0.0522 & 0.0506 & 0.0514 & 0.0534 & 0.0528 & 0.0442 & 0.0518 & 0.0446 & 0.051 \\
0 & 0.9 & 0.0528 & 0.053 & 0.0512 & 0.0524 & 0.0526 & 0.0768 & 0.0516 & 0.0458 & 0.0504 \\
0.5 & 0.1 & 0.4896 & 0.6472 & 0.6396 & 0.6306 & 0.631 & 0.1798 & 0.6438 & 0.338 & 0.4112 \\
0.5 & 0.5 & 0.5912 & 0.7412 & 0.734 & 0.7642 & 0.7608 & 0.343 & 0.7552 & 0.4686 & 0.5862 \\
0.5 & 0.9 & 0.9224 & 0.9334 & 0.9292 & 0.9796 & 0.9802 & 0.892 & 0.9542 & 0.9052 & 0.9746 \\
1.0 & 0.1 & 0.9004 & 0.9752 & 0.9736 & 0.978 & 0.9784 & 0.5504 & 0.9788 & 0.7466 & 0.8492 \\
1.0 & 0.5 & 0.948 & 0.9908 & 0.9902 & 0.9952 & 0.9954 & 0.8034 & 0.9926 & 0.8806 & 0.9526 \\
1.0 & 0.9 & 0.9978 & 0.999 & 0.999 & 1 & 1 & 0.9954 & 1 & 0.9958 & 1 \\
\hline
\end{tabular}

Table IV.10. Normal Distribution with $n_{p}=10, n_{u x}=5, n_{u y}=5$. Empirical Size and Power at $\alpha=0.05$

\begin{tabular}{ccccccccccc}
\hline$\delta$ & $\rho$ & $T$ & $R_{z}$ & $R_{\text {perm }}$ & $R_{w, z}$ & $R_{w, \text { perm }}$ & $Z_{b}$ & $M$ & $t$ & $S$ \\
\hline 0 & 0.1 & 0.054 & 0.0554 & 0.0468 & 0.052 & 0.0512 & 0.0746 & 0.0494 & 0.049 & 0.0514 \\
0 & 0.5 & 0.0554 & 0.0544 & 0.046 & 0.0492 & 0.0504 & 0.0884 & 0.0496 & 0.049 & 0.0514 \\
0 & 0.9 & 0.0522 & 0.0536 & 0.0466 & 0.0494 & 0.0504 & 0.1032 & 0.0498 & 0.049 & 0.0514 \\
0.5 & 0.1 & 0.3966 & 0.3788 & 0.345 & 0.3658 & 0.3648 & 0.2304 & 0.3516 & 0.291 & 0.2908 \\
0.5 & 0.5 & 0.5132 & 0.5068 & 0.466 & 0.489 & 0.4846 & 0.3844 & 0.4434 & 0.4254 & 0.4282 \\
0.5 & 0.9 & 0.9436 & 0.9288 & 0.9086 & 0.9114 & 0.9068 & 0.9416 & 0.7712 & 0.9436 & 0.938 \\
1.0 & 0.1 & 0.8566 & 0.84 & 0.8134 & 0.8298 & 0.829 & 0.593 & 0.8076 & 0.6956 & 0.689 \\
1.0 & 0.5 & 0.951 & 0.9482 & 0.9332 & 0.9414 & 0.9372 & 0.8564 & 0.899 & 0.8962 & 0.8872 \\
1.0 & 0.9 & 0.9998 & 1 & 0.9992 & 0.9994 & 0.999 & 1 & 0.97 & 1 & 1 \\
\hline
\end{tabular}


Table IV.11. Normal Distribution with $n_{p}=20, n_{u x}=10, n_{u y}=10$. Empirical Size and Power at $\alpha=0.05$

\begin{tabular}{ccccccccccc}
\hline$\delta$ & $\rho$ & $T$ & $R_{z}$ & $R_{\text {perm }}$ & $R_{w, z}$ & $R_{w, \text { perm }}$ & $Z_{b}$ & $M$ & $t$ & $S$ \\
\hline 0 & 0.1 & 0.0562 & 0.051 & 0.0496 & 0.0528 & 0.0514 & 0.06 & 0.0508 & 0.0454 & 0.0464 \\
0 & 0.5 & 0.0538 & 0.0472 & 0.0458 & 0.0486 & 0.0486 & 0.0762 & 0.0518 & 0.0454 & 0.0464 \\
0 & 0.9 & 0.049 & 0.0502 & 0.0494 & 0.0518 & 0.052 & 0.0964 & 0.0514 & 0.0454 & 0.0464 \\
0.5 & 0.1 & 0.6316 & 0.5936 & 0.5942 & 0.5996 & 0.6016 & 0.379 & 0.5854 & 0.4952 & 0.4722 \\
0.5 & 0.5 & 0.7858 & 0.761 & 0.759 & 0.7664 & 0.7668 & 0.6434 & 0.7184 & 0.702 & 0.6776 \\
0.5 & 0.9 & 0.999 & 0.9976 & 0.9972 & 0.9978 & 0.9972 & 0.9988 & 0.976 & 0.999 & 0.9978 \\
1.0 & 0.1 & 0.9892 & 0.984 & 0.9832 & 0.9846 & 0.9842 & 0.887 & 0.9806 & 0.9384 & 0.926 \\
1.0 & 0.5 & 0.999 & 0.9984 & 0.9988 & 0.9988 & 0.9988 & 0.9916 & 0.9966 & 0.9956 & 0.9928 \\
1.0 & 0.9 & 1 & 1 & 1 & 1 & 1 & 1 & 0.9998 & 1 & 1 \\
\hline
\end{tabular}

Table IV.12. Normal Distribution with $n_{p}=5, n_{u x}=7, n_{u y}=8$. Empirical Size and Power at $\alpha=0.05$

\begin{tabular}{ccccccccccc}
\hline$\delta$ & $\rho$ & $T$ & $R_{z}$ & $R_{\text {perm }}$ & $R_{w, z}$ & $R_{w, \text { perm }}$ & $Z_{b}$ & $M$ & $t$ & $S$ \\
\hline 0 & 0.1 & 0.062 & 0.06 & 0.0494 & 0.0504 & 0.0512 & 0.0434 & 0.0532 & 0.0472 & 0.0604 \\
0 & 0.5 & 0.0648 & 0.0598 & 0.0488 & 0.0542 & 0.0546 & 0.0638 & 0.057 & 0.0472 & 0.0604 \\
0 & 0.9 & 0.0616 & 0.0612 & 0.0502 & 0.0518 & 0.0546 & 0.0884 & 0.0556 & 0.0472 & 0.0604 \\
0.5 & 0.1 & 0.346 & 0.3188 & 0.2826 & 0.3158 & 0.3096 & 0.0806 & 0.3222 & 0.1686 & 0.1996 \\
0.5 & 0.5 & 0.406 & 0.352 & 0.3144 & 0.3728 & 0.3714 & 0.1434 & 0.3826 & 0.2358 & 0.2798 \\
0.5 & 0.9 & 0.7044 & 0.4688 & 0.4272 & 0.62 & 0.6174 & 0.5932 & 0.623 & 0.6608 & 0.713 \\
1.0 & 0.1 & 0.7818 & 0.7338 & 0.6954 & 0.7446 & 0.7412 & 0.164 & 0.7554 & 0.4024 & 0.4484 \\
1.0 & 0.5 & 0.8538 & 0.7786 & 0.7392 & 0.8366 & 0.8348 & 0.3554 & 0.8418 & 0.5776 & 0.6354 \\
1.0 & 0.9 & 0.9878 & 0.832 & 0.7998 & 0.9352 & 0.9362 & 0.9634 & 0.9352 & 0.9906 & 0.9942 \\
\hline
\end{tabular}




\section{CHAPTER V}

\section{DISCUSSION}

From the results of the simulations, several different tests stood out for certain cases, and it is hard to suggest a single best statistic. For the normal distributions, $T$ is most likely the best choice since it had the highest power much more often than any of the other tests. However, since a researcher will not likely know the true distribution in practice, and since the rank based statistics were most powerful for nonnormal distributions and nearly as powerful as $T$ for normal distributions, one of the rank based statistics will generally be the best choice. The light-tailed and heavy-tailed skewed distributions revealed that the weight on both $R_{w, z}$ and $R_{w, p e r m}$ was more important as the total sample sizes and percent missing increased. $R_{z}$ often had the highest power in the heavy-tailed symmetric case, along with $R_{w, z}$ and $R_{w, p e r m}$ as the sample size increased. Those statistics were also generally more powerful for low correlation, while $M$ usually had higher power for high correlation. With about $50 \%$ or fewer incomplete pairs and high correlation, $S$ was very competitive and often had the highest power for the nonnormal distributions. This suggests that we may not lose too much information by discarding the incomplete pairs in those cases. For small sample sizes, $R_{z}$ and $R_{w, z}$ often had higher power than the permutation versions, but this may be due to poor approximations for the asymptotic statistics as higher estimated Type I error rate estimates were also observed in these cases. Thus, the permutation statistics $R_{\text {perm }}$ and $R_{w, p e r m}$, which are guaranteed to provide unbiased power and Type I error rate estimates, should be preferred for small sample sizes. 


\section{BIBLIOGRAPHY}

[1] D. S. Bhoj. Testing equality of means of correlated variates with missing data on both responses. Biometrika, 65:225-228, 1978.

[2] D. S. Bhoj. On comparing correlated means in the presence of incomplete data. Biometrical Journal, 31:279-288, 1989.

[3] S. R. Dubnicka, R. C. Blair, and T. P. Hettmansperger. Rank-based procedures for mixed paired and two-sample designs. Journal of Modern Applied Statistical Methods, 1:32-41, 2002.

[4] R. L. Einsporn and D. Habtzghi. Combining paired and two-sample data using a permutation test. Journal of Data Science, 11:767-779, 2013.

[5] D. C. Hoaglin. Summarizing Shape Numerically: The g-and-h Distributions, chapter 11, pages 461-513. Wiley Series in Probability and Statistics. John Wiley \& Sons, Inc., Hoboken, New Jersey, 1985, 2006.

[6] P. E. Lin and L. E. Stivers. On the difference of means with incomplete data. Biometrika, 61:325-334, 1974.

[7] R. C. Magel and R. Fu. Proposed nonparametric test for the mixed two-sample design. Journal of Statistical Theory and Practice, 8:221-237, 2014.

[8] J. S. Maritz. A permutation paired test allowing for missing values. Australian Journal of Statistics, 37:153-159, 1995.

[9] M. Marozzi. Multivariate tests based on interpoint distances with application to magnetic resonance imaging. Statistical Methods in Medical Research, 25(6), 2014. 


\section{APPENDIX A}

\section{TABLES}

The following tables show supplementary simulation results.

Table A.1. Normal Distribution with $n_{p}=10, n_{u x}=0, n_{u y}=0$. Empirical Size and Power at $\alpha=0.05$

\begin{tabular}{ccccccccccc}
\hline$\delta$ & $\rho$ & $T$ & $R_{z}$ & $R_{\text {perm }}$ & $R_{w, z}$ & $R_{w, \text { perm }}$ & $Z_{b}$ & $M$ & $t$ & $S$ \\
\hline 0 & 0.1 & 0.0502 & 0.0526 & 0.0458 & 0.0526 & 0.0458 & 0.0492 & 0.0526 & 0.0492 & 0.0526 \\
0 & 0.5 & 0.0502 & 0.0526 & 0.0458 & 0.0526 & 0.0458 & 0.0492 & 0.0526 & 0.0492 & 0.0526 \\
0 & 0.9 & 0.0502 & 0.0422 & 0.0458 & 0.0422 & 0.0458 & 0.0492 & 0.0526 & 0.0492 & 0.0526 \\
0.5 & 0.1 & 0.2876 & 0.2926 & 0.2662 & 0.2926 & 0.2662 & 0.2842 & 0.2926 & 0.2842 & 0.2926 \\
0.5 & 0.5 & 0.423 & 0.4256 & 0.3936 & 0.4256 & 0.3936 & 0.4272 & 0.4256 & 0.4272 & 0.4256 \\
0.5 & 0.9 & 0.9466 & 0.9428 & 0.9278 & 0.9428 & 0.9278 & 0.9474 & 0.9428 & 0.9474 & 0.9428 \\
1.0 & 0.1 & 0.697 & 0.698 & 0.6634 & 0.698 & 0.6634 & 0.7002 & 0.698 & 0.7002 & 0.698 \\
1.0 & 0.5 & 0.8974 & 0.8902 & 0.869 & 0.8902 & 0.869 & 0.8992 & 0.8902 & 0.8992 & 0.8902 \\
1.0 & 0.9 & 1 & 1 & 1 & 1 & 1 & 1 & 1 & 1 & 1 \\
\hline
\end{tabular}

Table A.2. Normal Distribution with $n_{p}=5, n_{u x}=2, n_{u y}=3$. Empirical Size and Power at $\alpha=0.05$

\begin{tabular}{ccccccccccc}
\hline$\delta$ & $\rho$ & $T$ & $R_{z}$ & $R_{\text {perm }}$ & $R_{w, z}$ & $R_{w, \text { perm }}$ & $Z_{b}$ & $M$ & $t$ & $S$ \\
\hline 0 & 0.1 & 0.062 & 0.0436 & 0.0392 & 0.0504 & 0.0484 & 0.1006 & 0.0632 & 0.0546 & 0.068 \\
0 & 0.5 & 0.0602 & 0.0468 & 0.0428 & 0.0534 & 0.0508 & 0.1096 & 0.0652 & 0.0546 & 0.068 \\
0 & 0.9 & 0.0588 & 0.046 & 0.0412 & 0.0534 & 0.05 & 0.113 & 0.0648 & 0.0546 & 0.068 \\
0.5 & 0.1 & 0.2408 & 0.203 & 0.1836 & 0.222 & 0.215 & 0.1748 & 0.241 & 0.176 & 0.2076 \\
0.5 & 0.5 & 0.3046 & 0.2648 & 0.2428 & 0.2848 & 0.2772 & 0.2592 & 0.292 & 0.2456 & 0.2834 \\
0.5 & 0.9 & 0.6508 & 0.5606 & 0.5312 & 0.5712 & 0.5574 & 0.6792 & 0.5136 & 0.6564 & 0.702 \\
1.0 & 0.1 & 0.5712 & 0.4926 & 0.4628 & 0.5234 & 0.5064 & 0.365 & 0.544 & 0.4032 & 0.4544 \\
1.0 & 0.5 & 0.7028 & 0.6354 & 0.6068 & 0.6548 & 0.6444 & 0.5612 & 0.6488 & 0.5808 & 0.6312 \\
1.0 & 0.9 & 0.9712 & 0.8932 & 0.8832 & 0.8934 & 0.8832 & 0.9892 & 0.7828 & 0.992 & 0.994 \\
\hline
\end{tabular}


Table A.3. Normal Distribution with $n_{p}=20, n_{u x}=0, n_{u y}=0$. Empirical Size and Power at $\alpha=0.05$

\begin{tabular}{ccccccccccc}
\hline$\delta$ & $\rho$ & $T$ & $R_{z}$ & $R_{\text {perm }}$ & $R_{w, z}$ & $R_{w, p e r m}$ & $Z_{b}$ & $M$ & $t$ & $S$ \\
\hline 0 & 0.1 & 0.0514 & 0.05 & 0.0504 & 0.05 & 0.0504 & 0.0514 & 0.05 & 0.0514 & 0.05 \\
0 & 0.5 & 0.0514 & 0.05 & 0.0504 & 0.05 & 0.0504 & 0.0514 & 0.05 & 0.0514 & 0.05 \\
0 & 0.9 & 0.0514 & 0.05 & 0.0504 & 0.05 & 0.0504 & 0.0514 & 0.05 & 0.0514 & 0.05 \\
0.25 & 0.1 & 0.2 & 0.1886 & 0.189 & 0.1886 & 0.189 & 0.1984 & 0.1886 & 0.1984 & 0.1886 \\
0.25 & 0.5 & 0.2854 & 0.2698 & 0.269 & 0.2698 & 0.269 & 0.282 & 0.2698 & 0.282 & 0.2698 \\
0.25 & 0.9 & 0.7748 & 0.7538 & 0.751 & 0.7538 & 0.751 & 0.7756 & 0.7538 & 0.7756 & 0.7538 \\
0.5 & 0.1 & 0.4838 & 0.462 & 0.4642 & 0.462 & 0.4642 & 0.4822 & 0.462 & 0.4822 & 0.462 \\
0.5 & 0.5 & 0.6942 & 0.6678 & 0.6676 & 0.6678 & 0.6676 & 0.6908 & 0.6678 & 0.6908 & 0.6678 \\
0.5 & 0.9 & 0.9992 & 0.9978 & 0.998 & 0.9978 & 0.998 & 0.999 & 0.9978 & 0.999 & 0.9978 \\
1.0 & 0.1 & 0.9386 & 0.9268 & 0.9274 & 0.9268 & 0.9274 & 0.9376 & 0.9268 & 0.9376 & 0.9268 \\
1.0 & 0.5 & 0.996 & 0.994 & 0.9936 & 0.994 & 0.9936 & 0.9962 & 0.994 & 0.9962 & 0.994 \\
1.0 & 0.9 & 1 & 1 & 1 & 1 & 1 & 1 & 1 & 1 & 1 \\
\hline
\end{tabular}

Table A.4. Normal Distribution with $n_{p}=15, n_{u x}=2, n_{u y}=3$. Empirical Size and Power at $\alpha=0.05$

\begin{tabular}{ccccccccccc}
\hline$\delta$ & $\rho$ & $T$ & $R_{z}$ & $R_{\text {perm }}$ & $R_{w, z}$ & $R_{w, \text { perm }}$ & $Z_{b}$ & $M$ & $t$ & $S$ \\
\hline 0 & 0.1 & 0.051 & 0.0484 & 0.0498 & 0.0528 & 0.0512 & 0.11 & 0.0532 & 0.0494 & 0.054 \\
0 & 0.5 & 0.0524 & 0.0484 & 0.05 & 0.052 & 0.0512 & 0.112 & 0.0512 & 0.0494 & 0.054 \\
0 & 0.9 & 0.0512 & 0.0484 & 0.05 & 0.0498 & 0.0498 & 0.11 & 0.0488 & 0.0494 & 0.054 \\
0.5 & 0.1 & 0.4384 & 0.39 & 0.3858 & 0.4236 & 0.4154 & 0.395 & 0.3568 & 0.3862 & 0.3908 \\
0.5 & 0.5 & 0.6074 & 0.5632 & 0.56 & 0.5814 & 0.5784 & 0.5826 & 0.477 & 0.571 & 0.5672 \\
0.5 & 0.9 & 0.9912 & 0.991 & 0.9902 & 0.9884 & 0.986 & 0.9922 & 0.8408 & 0.9916 & 0.9922 \\
1.0 & 0.1 & 0.9016 & 0.8576 & 0.8564 & 0.8824 & 0.8784 & 0.8614 & 0.8046 & 0.8572 & 0.856 \\
1.0 & 0.5 & 0.9846 & 0.978 & 0.9774 & 0.9808 & 0.9798 & 0.9804 & 0.901 & 0.9794 & 0.9764 \\
1.0 & 0.9 & 1 & 1 & 1 & 1 & 1 & 1 & 0.9682 & 1 & 1 \\
\hline
\end{tabular}

Table A.5. Normal Distribution with $n_{p}=40, n_{u x}=0, n_{u y}=0$. Empirical Size and Power at $\alpha=0.05$

\begin{tabular}{ccccccccccc}
\hline$\delta$ & $\rho$ & $T$ & $R_{z}$ & $R_{\text {perm }}$ & $R_{w, z}$ & $R_{w, \text { perm }}$ & $Z_{b}$ & $M$ & $t$ & $S$ \\
\hline 0 & 0.1 & 0.0502 & 0.0468 & 0.0494 & 0.0468 & 0.0494 & 0.0488 & 0.0468 & 0.0488 & 0.0468 \\
0 & 0.5 & 0.0502 & 0.0468 & 0.0494 & 0.0468 & 0.0494 & 0.0488 & 0.0468 & 0.0488 & 0.0468 \\
0 & 0.9 & 0.0502 & 0.0468 & 0.0494 & 0.0468 & 0.0494 & 0.0488 & 0.0468 & 0.0488 & 0.0468 \\
0.25 & 0.1 & 0.325 & 0.3098 & 0.3054 & 0.3098 & 0.3054 & 0.3232 & 0.3098 & 0.3232 & 0.3098 \\
0.25 & 0.5 & 0.469 & 0.452 & 0.4498 & 0.452 & 0.4498 & 0.466 & 0.452 & 0.466 & 0.452 \\
0.25 & 0.9 & 0.9646 & 0.9598 & 0.9582 & 0.9598 & 0.9528 & 0.9652 & 0.9598 & 0.9652 & 0.9598 \\
0.5 & 0.1 & 0.7482 & 0.7284 & 0.725 & 0.7284 & 0.725 & 0.7436 & 0.7284 & 0.7436 & 0.7284 \\
0.5 & 0.5 & 0.929 & 0.9168 & 0.9166 & 0.9168 & 0.9166 & 0.9266 & 0.9168 & 0.9266 & 0.9168 \\
0.5 & 0.9 & 1 & 1 & 1 & 1 & 1 & 1 & 1 & 1 & 1 \\
1.0 & 0.1 & 0.999 & 0.998 & 0.9976 & 0.998 & 0.9976 & 0.9992 & 0.998 & 0.9992 & 0.998 \\
1.0 & 0.5 & 1 & 1 & 1 & 1 & 1 & 1 & 1 & 1 & 1 \\
1.0 & 0.9 & 1 & 1 & 1 & 1 & 1 & 1 & 1 & 1 & 1 \\
\hline
\end{tabular}


Table A.6. Normal Distribution with $n_{p}=32, n_{u x}=4, n_{u y}=4$. Empirical Size and Power at $\alpha=0.05$

\begin{tabular}{ccccccccccc}
\hline$\delta$ & $\rho$ & $T$ & $R_{z}$ & $R_{\text {perm }}$ & $R_{w, z}$ & $R_{w, \text { perm }}$ & $Z_{b}$ & $M$ & $t$ & $S$ \\
\hline 0 & 0.1 & 0.0524 & 0.0488 & 0.051 & 0.0506 & 0.0546 & 0.0962 & 0.0512 & 0.049 & 0.0506 \\
0 & 0.5 & 0.0528 & 0.0496 & 0.051 & 0.0496 & 0.051 & 0.1 & 0.0516 & 0.049 & 0.0506 \\
0 & 0.9 & 0.0532 & 0.0486 & 0.0504 & 0.049 & 0.0504 & 0.1024 & 0.053 & 0.049 & 0.0506 \\
0.5 & 0.1 & 0.7016 & 0.6516 & 0.6492 & 0.678 & 0.678 & 0.652 & 0.5904 & 0.657 & 0.6446 \\
0.5 & 0.5 & 0.887 & 0.8626 & 0.8642 & 0.8732 & 0.8736 & 0.8682 & 0.744 & 0.8706 & 0.8598 \\
0.5 & 0.9 & 1 & 1 & 1 & 1 & 1 & 1 & 0.9942 & 1 & 1 \\
1.0 & 0.1 & 0.9978 & 0.9934 & 0.993 & 0.9948 & 0.9942 & 0.9938 & 0.9774 & 0.9942 & 0.9922 \\
1.0 & 0.5 & 1 & 1 & 1 & 1 & 1 & 1 & 0.9976 & 1 & 1 \\
1.0 & 0.9 & 1 & 1 & 1 & 1 & 1 & 1 & 1 & 1 & 1 \\
\hline
\end{tabular}

Table A.7. Normal Distribution with $n_{p}=8, n_{u x}=16, n_{u y}=16$. Empirical Size and Power at $\alpha=0.05$

\begin{tabular}{ccccccccccc}
\hline$\delta$ & $\rho$ & $T$ & $R_{z}$ & $R_{\text {perm }}$ & $R_{w, z}$ & $R_{w, \text { perm }}$ & $Z_{b}$ & $M$ & $t$ & $S$ \\
\hline 0 & 0.1 & 0.0606 & 0.0512 & 0.0516 & 0.0516 & 0.0526 & 0.024 & 0.052 & 0.047 & 0.0514 \\
0 & 0.5 & 0.0608 & 0.0532 & 0.054 & 0.0528 & 0.0526 & 0.0466 & 0.0522 & 0.047 & 0.0514 \\
0 & 0.9 & 0.058 & 0.0496 & 0.0514 & 0.0514 & 0.0534 & 0.082 & 0.0514 & 0.047 & 0.0514 \\
0.5 & 0.1 & 0.5448 & 0.4562 & 0.4552 & 0.502 & 0.5028 & 0.059 & 0.501 & 0.2418 & 0.2554 \\
0.5 & 0.5 & 0.618 & 0.4928 & 0.4892 & 0.5892 & 0.5924 & 0.1618 & 0.592 & 0.362 & 0.3692 \\
0.5 & 0.9 & 0.917 & 0.5908 & 0.5892 & 0.8604 & 0.862 & 0.8188 & 0.868 & 0.8826 & 0.8856 \\
1.0 & 0.1 & 0.9628 & 0.9254 & 0.9206 & 0.95 & 0.9508 & 0.189 & 0.949 & 0.6096 & 0.6252 \\
1.0 & 0.5 & 0.9838 & 0.9422 & 0.9394 & 0.983 & 0.9826 & 0.4926 & 0.9826 & 0.8142 & 0.816 \\
1.0 & 0.9 & 1 & 0.9538 & 0.9536 & 0.997 & 0.9968 & 0.9982 & 0.9974 & 1 & 1 \\
\hline
\end{tabular}

Table A.8. Normal Distribution with $n_{p}=10, n_{u x}=5, n_{u y}=10$. Empirical Size and Power at $\alpha=0.05$

\begin{tabular}{ccccccccccc}
\hline$\delta$ & $\rho$ & $T$ & $R_{z}$ & $R_{\text {perm }}$ & $R_{w, z}$ & $R_{w, \text { perm }}$ & $Z_{b}$ & $M$ & $t$ & $S$ \\
\hline 0 & 0.1 & 0.061 & 0.0558 & 0.0512 & 0.0542 & 0.0542 & 0.0566 & 0.057 & 0.0476 & 0.053 \\
0 & 0.5 & 0.0616 & 0.0578 & 0.053 & 0.0556 & 0.0564 & 0.0736 & 0.0588 & 0.0476 & 0.053 \\
0 & 0.9 & 0.0594 & 0.0584 & 0.0548 & 0.0566 & 0.057 & 0.0954 & 0.0588 & 0.0476 & 0.053 \\
0.5 & 0.1 & 0.4352 & 0.4072 & 0.3916 & 0.3998 & 0.4002 & 0.1858 & 0.4046 & 0.2858 & 0.2892 \\
0.5 & 0.5 & 0.5456 & 0.5086 & 0.4934 & 0.52 & 0.5236 & 0.342 & 0.4968 & 0.4164 & 0.413 \\
0.5 & 0.9 & 0.9488 & 0.8648 & 0.8526 & 0.912 & 0.9082 & 0.9362 & 0.8206 & 0.9438 & 0.94 \\
1.0 & 0.1 & 0.8982 & 0.8728 & 0.863 & 0.87 & 0.8704 & 0.5108 & 0.8664 & 0.704 & 0.6964 \\
1.0 & 0.5 & 0.9648 & 0.9472 & 0.941 & 0.9548 & 0.9538 & 0.8284 & 0.9332 & 0.9002 & 0.8914 \\
1.0 & 0.9 & 1 & 0.9918 & 0.99 & 0.9982 & 0.9982 & 1 & 0.9822 & 1 & 1 \\
\hline
\end{tabular}


Table A.9. Normal Distribution with $n_{p}=20, n_{u x}=30, n_{u y}=10$. Empirical Size and Power at $\alpha=0.05$

\begin{tabular}{ccccccccccc}
\hline$\delta$ & $\rho$ & $T$ & $R_{z}$ & $R_{\text {perm }}$ & $R_{w, z}$ & $R_{w, \text { perm }}$ & $Z_{b}$ & $M$ & $t$ & $S$ \\
\hline 0 & 0.1 & 0.0542 & 0.0488 & 0.0476 & 0.05 & 0.0544 & 0.0348 & 0.049 & 0.0512 & 0.0496 \\
0 & 0.5 & 0.0564 & 0.0502 & 0.0496 & 0.0502 & 0.0524 & 0.0546 & 0.0512 & 0.0512 & 0.0496 \\
0 & 0.9 & 0.0544 & 0.052 & 0.0504 & 0.0504 & 0.0542 & 0.0878 & 0.0512 & 0.0512 & 0.0496 \\
0.5 & 0.1 & 0.6918 & 0.6464 & 0.6414 & 0.6516 & 0.6516 & 0.2778 & 0.6546 & 0.48 & 0.464 \\
0.5 & 0.5 & 0.8274 & 0.763 & 0.758 & 0.8124 & 0.8108 & 0.5606 & 0.783 & 0.6864 & 0.6676 \\
0.5 & 0.9 & 0.9994 & 0.9698 & 0.9686 & 0.998 & 0.9984 & 0.9994 & 0.985 & 0.9994 & 0.999 \\
1.0 & 0.1 & 0.996 & 0.9924 & 0.991 & 0.9948 & 0.9942 & 0.8274 & 0.9944 & 0.9462 & 0.9386 \\
1.0 & 0.5 & 0.9996 & 0.998 & 0.9974 & 0.9996 & 0.9994 & 0.9888 & 0.9986 & 0.9968 & 0.9956 \\
1.0 & 0.9 & 1 & 1 & 1 & 1 & 1 & 1 & 1 & 1 & 1 \\
\hline
\end{tabular}

Table A.10. Light-Tailed Skewed Distribution with $n_{p}=10, n_{u x}=0, n_{u y}=0$. Empirical Size and Power at $\alpha=0.05$

\begin{tabular}{ccccccccccc}
\hline$\delta$ & $\rho$ & $T$ & $R_{z}$ & $R_{\text {perm }}$ & $R_{w, z}$ & $R_{w, \text { perm }}$ & $Z_{b}$ & $M$ & $t$ & $S$ \\
\hline 0 & 0.1 & 0.0516 & 0.0416 & 0.0438 & 0.0416 & 0.0438 & 0.0458 & 0.0516 & 0.0458 & 0.0516 \\
0 & 0.5 & 0.051 & 0.051 & 0.0448 & 0.051 & 0.0448 & 0.045 & 0.051 & 0.045 & 0.051 \\
0 & 0.9 & 0.0508 & 0.0508 & 0.0456 & 0.0508 & 0.0456 & 0.0446 & 0.0508 & 0.0446 & 0.0508 \\
0.5 & 0.1 & 0.2452 & 0.2684 & 0.2418 & 0.2684 & 0.2418 & 0.232 & 0.2684 & 0.232 & 0.2684 \\
0.5 & 0.5 & 0.346 & 0.389 & 0.354 & 0.389 & 0.354 & 0.3276 & 0.389 & 0.3276 & 0.389 \\
0.5 & 0.9 & 0.7576 & 0.8152 & 0.7854 & 0.8152 & 0.7854 & 0.7484 & 0.8152 & 0.7484 & 0.8152 \\
1.0 & 0.1 & 0.5434 & 0.5992 & 0.5632 & 0.5992 & 0.5632 & 0.5308 & 0.5992 & 0.5308 & 0.5992 \\
1.0 & 0.5 & 0.7138 & 0.7672 & 0.7346 & 0.7672 & 0.7346 & 0.702 & 0.7672 & 0.702 & 0.7672 \\
1.0 & 0.9 & 0.9706 & 0.9872 & 0.9786 & 0.9872 & 0.9786 & 0.9706 & 0.9872 & 0.9706 & 0.9872 \\
\hline
\end{tabular}

Table A.11. Light-Tailed Skewed Distribution with $n_{p}=5, n_{u x}=2, n_{u y}=3$. Empirical Size and Power at $\alpha=0.05$

\begin{tabular}{ccccccccccc}
\hline$\delta$ & $\rho$ & $T$ & $R_{z}$ & $R_{\text {perm }}$ & $R_{w, z}$ & $R_{w, \text { perm }}$ & $Z_{b}$ & $M$ & $t$ & $S$ \\
\hline 0 & 0.1 & 0.0624 & 0.0442 & 0.039 & 0.0504 & 0.0488 & 0.081 & 0.0602 & 0.0428 & 0.0654 \\
0 & 0.5 & 0.0612 & 0.0474 & 0.0418 & 0.0538 & 0.0508 & 0.0866 & 0.0628 & 0.041 & 0.065 \\
0 & 0.9 & 0.0568 & 0.047 & 0.0418 & 0.054 & 0.0508 & 0.0872 & 0.062 & 0.0388 & 0.0652 \\
0.5 & 0.1 & 0.2292 & 0.205 & 0.1906 & 0.2278 & 0.2196 & 0.1562 & 0.2482 & 0.1562 & 0.2184 \\
0.5 & 0.5 & 0.2844 & 0.2692 & 0.2522 & 0.292 & 0.2796 & 0.228 & 0.3032 & 0.2206 & 0.298 \\
0.5 & 0.9 & 0.5462 & 0.5104 & 0.4832 & 0.5214 & 0.4832 & 0.5536 & 0.486 & 0.539 & 0.6132 \\
1.0 & 0.1 & 0.474 & 0.4602 & 0.4314 & 0.4922 & 0.475 & 0.3268 & 0.522 & 0.353 & 0.4358 \\
1.0 & 0.5 & 0.5728 & 0.5658 & 0.541 & 0.5884 & 0.5744 & 0.4662 & 0.6058 & 0.4912 & 0.5618 \\
1.0 & 0.9 & 0.845 & 0.8138 & 0.7878 & 0.8222 & 0.8048 & 0.8628 & 0.7702 & 0.8614 & 0.8876 \\
\hline
\end{tabular}


Table A.12. Light-Tailed Skewed Distribution with $n_{p}=20, n_{u x}=0, n_{u y}=0$. Empirical Size and Power at $\alpha=0.05$

\begin{tabular}{ccccccccccc}
\hline$\delta$ & $\rho$ & $T$ & $R_{z}$ & $R_{\text {perm }}$ & $R_{w, z}$ & $R_{w, \text { perm }}$ & $Z_{b}$ & $M$ & $t$ & $S$ \\
\hline 0 & 0.1 & 0.0496 & 0.0474 & 0.0496 & 0.0474 & 0.0496 & 0.045 & 0.0474 & 0.045 & 0.0474 \\
0 & 0.5 & 0.0498 & 0.0478 & 0.05 & 0.0478 & 0.05 & 0.0442 & 0.0478 & 0.0442 & 0.0478 \\
0 & 0.9 & 0.05 & 0.049 & 0.0512 & 0.049 & 0.0512 & 0.045 & 0.049 & 0.045 & 0.049 \\
0.5 & 0.1 & 0.3496 & 0.405 & 0.4076 & 0.405 & 0.4076 & 0.3394 & 0.405 & 0.3394 & 0.405 \\
0.5 & 0.5 & 0.4846 & 0.5896 & 0.587 & 0.5896 & 0.587 & 0.478 & 0.5896 & 0.478 & 0.5896 \\
0.5 & 0.9 & 0.904 & 0.9654 & 0.9646 & 0.9654 & 0.9646 & 0.9026 & 0.9654 & 0.9026 & 0.9654 \\
1.0 & 0.1 & 0.752 & 0.8446 & 0.8412 & 0.8446 & 0.8412 & 0.752 & 0.8446 & 0.752 & 0.8446 \\
1.0 & 0.5 & 0.8806 & 0.9456 & 0.9448 & 0.9456 & 0.9448 & 0.878 & 0.9456 & 0.878 & 0.9456 \\
1.0 & 0.9 & 0.9966 & 0.9998 & 0.9998 & 0.9998 & 0.9998 & 0.9962 & 0.9998 & 0.9962 & 0.9998 \\
\hline
\end{tabular}

Table A.13. Light-Tailed Skewed Distribution with $n_{p}=15, n_{u x}=2, n_{u y}=3$. Empirical Size and Power at $\alpha=0.05$

\begin{tabular}{ccccccccccc}
\hline$\delta$ & $\rho$ & $T$ & $R_{z}$ & $R_{\text {perm }}$ & $R_{w, z}$ & $R_{w, \text { perm }}$ & $Z_{b}$ & $M$ & $t$ & $S$ \\
\hline 0 & 0.1 & 0.0526 & 0.05 & 0.0508 & 0.053 & 0.05 & 0.1038 & 0.0532 & 0.0462 & 0.0554 \\
0 & 0.5 & 0.0538 & 0.0496 & 0.0492 & 0.052 & 0.0514 & 0.1046 & 0.054 & 0.0438 & 0.0554 \\
0 & 0.9 & 0.0522 & 0.049 & 0.0506 & 0.0526 & 0.0506 & 0.0978 & 0.0494 & 0.044 & 0.0534 \\
0.5 & 0.1 & 0.327 & 0.3542 & 0.352 & 0.3834 & 0.3796 & 0.2992 & 0.3534 & 0.297 & 0.3586 \\
0.5 & 0.5 & 0.4358 & 0.4972 & 0.494 & 0.5228 & 0.5166 & 0.4164 & 0.4514 & 0.4036 & 0.5008 \\
0.5 & 0.9 & 0.8474 & 0.9166 & 0.9136 & 0.9154 & 0.9142 & 0.8514 & 0.7602 & 0.8444 & 0.9216 \\
1.0 & 0.1 & 0.7006 & 0.757 & 0.755 & 0.7996 & 0.7952 & 0.6484 & 0.7474 & 0.6506 & 0.7466 \\
1.0 & 0.5 & 0.8324 & 0.8946 & 0.8928 & 0.9128 & 0.9102 & 0.8124 & 0.8424 & 0.8084 & 0.8914 \\
1.0 & 0.9 & 0.9904 & 0.9978 & 0.9974 & 0.9976 & 0.9974 & 0.99 & 0.9564 & 0.9892 & 0.9978 \\
\hline
\end{tabular}

Table A.14. Light-Tailed Skewed Distribution with $n_{p}=40, n_{u x}=0, n_{u y}=0$. Empirical Size and Power at $\alpha=0.05$

\begin{tabular}{ccccccccccc}
\hline$\delta$ & $\rho$ & $T$ & $R_{z}$ & $R_{\text {perm }}$ & $R_{w, z}$ & $R_{w, \text { perm }}$ & $Z_{b}$ & $M$ & $t$ & $S$ \\
\hline 0 & 0.1 & 0.0542 & 0.0514 & 0.051 & 0.0514 & 0.051 & 0.0532 & 0.0514 & 0.0532 & 0.0514 \\
0 & 0.5 & 0.0548 & 0.0512 & 0.0508 & 0.0521 & 0.0508 & 0.0538 & 0.0512 & 0.0538 & 0.0512 \\
0 & 0.9 & 0.056 & 0.051 & 0.0514 & 0.051 & 0.0514 & 0.0526 & 0.051 & 0.0526 & 0.051 \\
0.5 & 0.1 & 0.5212 & 0.6582 & 0.6548 & 0.6582 & 0.6548 & 0.519 & 0.6582 & 0.519 & 0.6582 \\
0.5 & 0.5 & 0.6912 & 0.853 & 0.851 & 0.853 & 0.851 & 0.686 & 0.853 & 0.686 & 0.853 \\
0.5 & 0.9 & 0.985 & 0.9994 & 0.9992 & 0.9994 & 0.9992 & 0.9846 & 0.9994 & 0.9846 & 0.9994 \\
1.0 & 0.1 & 0.9258 & 0.9826 & 0.982 & 0.9826 & 0.982 & 0.9228 & 0.9826 & 0.9228 & 0.9826 \\
1.0 & 0.5 & 0.981 & 0.9984 & 0.9982 & 0.9984 & 0.9982 & 0.9796 & 0.9984 & 0.9796 & 0.9984 \\
1.0 & 0.9 & 0.9998 & 1 & 1 & 1 & 1 & 0.9998 & 1 & 0.9998 & 1 \\
\hline
\end{tabular}


Table A.15. Light-Tailed Skewed Distribution with $n_{p}=32, n_{u x}=4, n_{u y}=4$. Empirical Size and Power at $\alpha=0.05$

\begin{tabular}{ccccccccccc}
\hline$\delta$ & $\rho$ & $T$ & $R_{z}$ & $R_{\text {perm }}$ & $R_{w, z}$ & $R_{w, \text { perm }}$ & $Z_{b}$ & $M$ & $t$ & $S$ \\
\hline 0 & 0.1 & 0.054 & 0.0502 & 0.0492 & 0.0526 & 0.053 & 0.0978 & 0.0504 & 0.053 & 0.051 \\
0 & 0.5 & 0.0544 & 0.0502 & 0.0498 & 0.0484 & 0.0526 & 0.1004 & 0.052 & 0.049 & 0.0492 \\
0 & 0.9 & 0.0508 & 0.0488 & 0.0498 & 0.0502 & 0.0524 & 0.1024 & 0.0522 & 0.0492 & 0.0504 \\
0.5 & 0.1 & 0.488 & 0.5926 & 0.595 & 0.6226 & 0.6214 & 0.4518 & 0.5616 & 0.4596 & 0.5824 \\
0.5 & 0.5 & 0.645 & 0.7838 & 0.7824 & 0.8034 & 0.8032 & 0.6198 & 0.6958 & 0.6214 & 0.777 \\
0.5 & 0.9 & 0.9696 & 0.9976 & 0.9978 & 0.9976 & 0.9978 & 0.9694 & 0.9666 & 0.969 & 0.998 \\
1.0 & 0.1 & 0.9028 & 0.966 & 0.965 & 0.9774 & 0.9766 & 0.8758 & 0.9474 & 0.8806 & 0.9612 \\
1.0 & 0.5 & 0.9662 & 0.9946 & 0.9946 & 0.9966 & 0.9964 & 0.9574 & 0.9844 & 0.9588 & 0.9934 \\
1.0 & 0.9 & 0.9992 & 1 & 1 & 1 & 1 & 0.9992 & 0.9994 & 0.9992 & 1 \\
\hline
\end{tabular}

Table A.16. Light-Tailed Skewed Distribution with $n_{p}=8, n_{u x}=16, n_{u y}=16$. Empirical Size and Power at $\alpha=0.05$

\begin{tabular}{ccccccccccc}
\hline$\delta$ & $\rho$ & $T$ & $R_{z}$ & $R_{\text {perm }}$ & $R_{w, z}$ & $R_{w, \text { perm }}$ & $Z_{b}$ & $M$ & $t$ & $S$ \\
\hline 0 & 0.1 & 0.0574 & 0.0512 & 0.0518 & 0.0538 & 0.0546 & 0.015 & 0.0534 & 0.0392 & 0.054 \\
0 & 0.5 & 0.0588 & 0.0524 & 0.052 & 0.0528 & 0.0526 & 0.028 & 0.0528 & 0.0388 & 0.053 \\
0 & 0.9 & 0.0576 & 0.051 & 0.0522 & 0.0532 & 0.0542 & 0.0574 & 0.0532 & 0.0372 & 0.0524 \\
0.5 & 0.1 & 0.3866 & 0.4974 & 0.4934 & 0.5198 & 0.52 & 0.046 & 0.5168 & 0.2126 & 0.2452 \\
0.5 & 0.5 & 0.4444 & 0.53 & 0.5272 & 0.6016 & 0.602 & 0.1246 & 0.6 & 0.296 & 0.3394 \\
0.5 & 0.9 & 0.7092 & 0.6124 & 0.6102 & 0.819 & 0.8188 & 0.5966 & 0.824 & 0.6782 & 0.698 \\
1.0 & 0.1 & 0.7916 & 0.9162 & 0.9142 & 0.9236 & 0.9236 & 0.146 & 0.921 & 0.4742 & 0.5068 \\
1.0 & 0.5 & 0.8358 & 0.9296 & 0.9298 & 0.9594 & 0.9574 & 0.3416 & 0.9594 & 0.6334 & 0.65 \\
1.0 & 0.9 & 0.963 & 0.9474 & 0.946 & 0.9922 & 0.9912 & 0.9078 & 0.9926 & 0.944 & 0.932 \\
\hline
\end{tabular}

Table A.17. Light-Tailed Skewed Distribution with $n_{p}=10, n_{u x}=5, n_{u y}=10$. Empirical Size and Power at $\alpha=0.05$

\begin{tabular}{ccccccccccc}
\hline$\delta$ & $\rho$ & $T$ & $R_{z}$ & $R_{\text {perm }}$ & $R_{w, z}$ & $R_{w, \text { perm }}$ & $Z_{b}$ & $M$ & $t$ & $S$ \\
\hline 0 & 0.1 & 0.0564 & 0.0536 & 0.049 & 0.0518 & 0.0508 & 0.0408 & 0.054 & 0.045 & 0.0524 \\
0 & 0.5 & 0.0604 & 0.054 & 0.0512 & 0.0538 & 0.0548 & 0.0548 & 0.0546 & 0.044 & 0.0514 \\
0 & 0.9 & 0.0584 & 0.058 & 0.0548 & 0.0568 & 0.057 & 0.0814 & 0.0584 & 0.0416 & 0.052 \\
0.5 & 0.1 & 0.3198 & 0.403 & 0.3888 & 0.3908 & 0.3928 & 0.1414 & 0.402 & 0.2322 & 0.274 \\
0.5 & 0.5 & 0.402 & 0.5012 & 0.482 & 0.4974 & 0.4972 & 0.2552 & 0.49 & 0.334 & 0.386 \\
0.5 & 0.9 & 0.747 & 0.7944 & 0.7768 & 0.8276 & 0.8282 & 0.7268 & 0.7618 & 0.7444 & 0.808 \\
1.0 & 0.1 & 0.6776 & 0.8244 & 0.814 & 0.8102 & 0.8084 & 0.3828 & 0.8276 & 0.5366 & 0.598 \\
1.0 & 0.5 & 0.779 & 0.9036 & 0.8956 & 0.9008 & 0.8988 & 0.6068 & 0.9018 & 0.6934 & 0.7554 \\
1.0 & 0.9 & 0.9732 & 0.9878 & 0.9866 & 0.9926 & 0.9922 & 0.965 & 0.9788 & 0.9688 & 0.9842 \\
\hline
\end{tabular}


Table A.18. Heavy-Tailed Skewed Distribution with $n_{p}=10, n_{u x}=0, n_{u y}=0$. Empirical Size and Power at $\alpha=0.05$

\begin{tabular}{ccccccccccc}
\hline$\delta$ & $\rho$ & $T$ & $R_{z}$ & $R_{\text {perm }}$ & $R_{w, z}$ & $R_{w, \text { perm }}$ & $Z_{b}$ & $M$ & $t$ & $S$ \\
\hline 0 & 0.1 & 0.049 & 0.0516 & 0.0458 & 0.0516 & 0.0458 & 0.0364 & 0.0516 & 0.0364 & 0.0516 \\
0 & 0.5 & 0.0476 & 0.0512 & 0.0434 & 0.0512 & 0.0434 & 0.0356 & 0.0512 & 0.0356 & 0.0512 \\
0 & 0.9 & 0.0486 & 0.0516 & 0.0442 & 0.0516 & 0.0442 & 0.0342 & 0.0516 & 0.0342 & 0.0516 \\
0.5 & 0.1 & 0.17 & 0.1912 & 0.1702 & 0.1912 & 0.1702 & 0.1392 & 0.1912 & 0.1392 & 0.1912 \\
0.5 & 0.5 & 0.2252 & 0.255 & 0.2306 & 0.255 & 0.2306 & 0.1824 & 0.255 & 0.1824 & 0.255 \\
0.5 & 0.9 & 0.5164 & 0.6002 & 0.5592 & 0.6002 & 0.5592 & 0.4542 & 0.6002 & 0.4542 & 0.6002 \\
1.0 & 0.1 & 0.3564 & 0.4168 & 0.3788 & 0.4168 & 0.3788 & 0.3024 & 0.4168 & 0.3024 & 0.4168 \\
1.0 & 0.5 & 0.4716 & 0.5474 & 0.5098 & 0.5474 & 0.5098 & 0.415 & 0.5474 & 0.415 & 0.5474 \\
1.0 & 0.9 & 0.7874 & 0.8974 & 0.8596 & 0.8974 & 0.8596 & 0.7502 & 0.8974 & 0.7502 & 0.8974 \\
\hline
\end{tabular}

Table A.19. Heavy-Tailed Skewed Distribution with $n_{p}=5, n_{u x}=2, n_{u y}=3$. Empirical Size and Power at $\alpha=0.05$

\begin{tabular}{ccccccccccc}
\hline$\delta$ & $\rho$ & $T$ & $R_{z}$ & $R_{\text {perm }}$ & $R_{w, z}$ & $R_{w, \text { perm }}$ & $Z_{b}$ & $M$ & $t$ & $S$ \\
\hline 0 & 0.1 & 0.0612 & 0.0442 & 0.0384 & 0.0506 & 0.0484 & 0.068 & 0.0604 & 0.0338 & 0.066 \\
0 & 0.5 & 0.0598 & 0.048 & 0.043 & 0.0536 & 0.0514 & 0.075 & 0.0654 & 0.0332 & 0.0664 \\
0 & 0.9 & 0.059 & 0.0484 & 0.0424 & 0.0542 & 0.0524 & 0.0766 & 0.0632 & 0.033 & 0.0668 \\
0.5 & 0.1 & 0.1718 & 0.1538 & 0.1424 & 0.1704 & 0.163 & 0.1082 & 0.1886 & 0.105 & 0.1702 \\
0.5 & 0.5 & 0.1996 & 0.1886 & 0.1728 & 0.204 & 0.1968 & 0.1482 & 0.2222 & 0.1358 & 0.216 \\
0.5 & 0.9 & 0.383 & 0.3606 & 0.339 & 0.3754 & 0.3652 & 0.3582 & 0.3588 & 0.3396 & 0.4546 \\
1.0 & 0.1 & 0.328 & 0.3178 & 0.299 & 0.3456 & 0.3326 & 0.2106 & 0.3786 & 0.2206 & 0.3176 \\
1.0 & 0.5 & 0.4028 & 0.396 & 0.3732 & 0.4222 & 0.4078 & 0.3006 & 0.4426 & 0.2986 & 0.4114 \\
1.0 & 0.9 & 0.6546 & 0.6444 & 0.6194 & 0.655 & 0.6382 & 0.6584 & 0.6128 & 0.6466 & 0.7294 \\
\hline
\end{tabular}

Table A.20. Heavy-Tailed Skewed Distribution with $n_{p}=20, n_{u x}=0, n_{u y}=0$. Empirical Size and Power at $\alpha=0.05$

\begin{tabular}{ccccccccccc}
\hline$\delta$ & $\rho$ & $T$ & $R_{z}$ & $R_{\text {perm }}$ & $R_{w, z}$ & $R_{w, \text { perm }}$ & $Z_{b}$ & $M$ & $t$ & $S$ \\
\hline 0 & 0.1 & 0.0512 & 0.0494 & 0.0522 & 0.0494 & 0.0522 & 0.0414 & 0.0494 & 0.0414 & 0.0494 \\
0 & 0.5 & 0.0512 & 0.0504 & 0.0534 & 0.0504 & 0.0534 & 0.0382 & 0.0504 & 0.0382 & 0.0504 \\
0 & 0.9 & 0.0526 & 0.049 & 0.054 & 0.049 & 0.054 & 0.038 & 0.049 & 0.038 & 0.049 \\
0.5 & 0.1 & 0.2058 & 0.2776 & 0.2724 & 0.2776 & 0.2724 & 0.1774 & 0.2776 & 0.1774 & 0.2776 \\
0.5 & 0.5 & 0.2738 & 0.3854 & 0.386 & 0.3854 & 0.386 & 0.2366 & 0.3854 & 0.2366 & 0.3854 \\
0.5 & 0.9 & 0.5962 & 0.844 & 0.8422 & 0.844 & 0.8422 & 0.548 & 0.8422 & 0.548 & 0.8422 \\
1.0 & 0.1 & 0.439 & 0.6224 & 0.6252 & 0.6224 & 0.6252 & 0.3974 & 0.6224 & 0.3974 & 0.6224 \\
1.0 & 0.5 & 0.5626 & 0.7842 & 0.7868 & 0.7842 & 0.7868 & 0.515 & 0.7842 & 0.515 & 0.7842 \\
1.0 & 0.9 & 0.8342 & 0.986 & 0.9844 & 0.986 & 0.9844 & 0.8176 & 0.986 & 0.8176 & 0.986 \\
\hline
\end{tabular}


Table A.21. Heavy-Tailed Skewed Distribution with $n_{p}=15, n_{u x}=2, n_{u y}=3$. Empirical Size and Power at $\alpha=0.05$

\begin{tabular}{ccccccccccc}
\hline$\delta$ & $\rho$ & $T$ & $R_{z}$ & $R_{\text {perm }}$ & $R_{w, z}$ & $R_{w, \text { perm }}$ & $Z_{b}$ & $M$ & $t$ & $S$ \\
\hline 0 & 0.1 & 0.051 & 0.049 & 0.0494 & 0.0518 & 0.0498 & 0.0872 & 0.0518 & 0.0398 & 0.0538 \\
0 & 0.5 & 0.0504 & 0.0492 & 0.0496 & 0.051 & 0.0508 & 0.0842 & 0.0536 & 0.0374 & 0.0542 \\
0 & 0.9 & 0.0528 & 0.0486 & 0.049 & 0.051 & 0.0514 & 0.0812 & 0.051 & 0.0368 & 0.0548 \\
0.5 & 0.1 & 0.1998 & 0.246 & 0.2402 & 0.2636 & 0.2616 & 0.1724 & 0.2522 & 0.1638 & 0.2492 \\
0.5 & 0.5 & 0.2562 & 0.3334 & 0.3306 & 0.3524 & 0.3466 & 0.2354 & 0.3114 & 0.2208 & 0.341 \\
0.5 & 0.9 & 0.5582 & 0.7528 & 0.7478 & 0.7512 & 0.7448 & 0.5204 & 0.5822 & 0.511 & 0.7616 \\
1.0 & 0.1 & 0.4166 & 0.5452 & 0.5428 & 0.591 & 0.5852 & 0.3594 & 0.5528 & 0.361 & 0.5364 \\
1.0 & 0.5 & 0.5292 & 0.7028 & 0.699 & 0.737 & 0.7326 & 0.4764 & 0.6588 & 0.4694 & 0.6958 \\
1.0 & 0.9 & 0.8122 & 0.9586 & 0.9578 & 0.959 & 0.958 & 0.7914 & 0.868 & 0.7872 & 0.9604 \\
\hline
\end{tabular}

Table A.22. Heavy-Tailed Skewed Distribution with $n_{p}=40, n_{u x}=0, n_{u y}=0$. Empirical Size and Power at $\alpha=0.05$

\begin{tabular}{ccccccccccc}
\hline$\delta$ & $\rho$ & $T$ & $R_{z}$ & $R_{\text {perm }}$ & $R_{w, z}$ & $R_{w, \text { perm }}$ & $Z_{b}$ & $M$ & $t$ & $S$ \\
\hline 0 & 0.1 & 0.054 & 0.0486 & 0.0492 & 0.0486 & 0.0492 & 0.0442 & 0.0486 & 0.0442 & 0.0486 \\
0 & 0.5 & 0.0458 & 0.0486 & 0.048 & 0.0486 & 0.048 & 0.0428 & 0.0486 & 0.0428 & 0.0486 \\
0 & 0.9 & 0.054 & 0.0492 & 0.0488 & 0.0492 & 0.0488 & 0.0408 & 0.0492 & 0.0408 & 0.0492 \\
0.5 & 0.1 & 0.258 & 0.4592 & 0.4552 & 0.4592 & 0.4552 & 0.238 & 0.4592 & 0.238 & 0.4592 \\
0.5 & 0.5 & 0.3448 & 0.622 & 0.6216 & 0.622 & 0.6216 & 0.3086 & 0.622 & 0.3086 & 0.622 \\
0.5 & 0.9 & 0.6732 & 0.9852 & 0.984 & 0.9852 & 0.984 & 0.6408 & 0.9852 & 0.6408 & 0.9852 \\
1.0 & 0.1 & 0.541 & 0.8798 & 0.8788 & 0.8798 & 0.8788 & 0.5112 & 0.8798 & 0.5112 & 0.8798 \\
1.0 & 0.5 & 0.6598 & 0.9696 & 0.9696 & 0.9696 & 0.9696 & 0.629 & 0.9696 & 0.629 & 0.9696 \\
1.0 & 0.9 & 0.8862 & 0.9998 & 0.9998 & 0.9998 & 0.9998 & 0.8722 & 0.9998 & 0.8722 & 0.9998 \\
\hline
\end{tabular}

Table A.23. Heavy-Tailed Skewed Distribution with $n_{p}=32, n_{u x}=4, n_{u y}=4$. Empirical Size and Power at $\alpha=0.05$

\begin{tabular}{ccccccccccc}
\hline$\delta$ & $\rho$ & $T$ & $R_{z}$ & $R_{\text {perm }}$ & $R_{w, z}$ & $R_{w, \text { perm }}$ & $Z_{b}$ & $M$ & $t$ & $S$ \\
\hline 0 & 0.1 & 0.052 & 0.0518 & 0.0496 & 0.0524 & 0.0522 & 0.077 & 0.0484 & 0.0426 & 0.0522 \\
0 & 0.5 & 0.0546 & 0.0506 & 0.0514 & 0.0524 & 0.0536 & 0.0802 & 0.0524 & 0.0398 & 0.052 \\
0 & 0.9 & 0.0554 & 0.0494 & 0.0506 & 0.0526 & 0.0544 & 0.082 & 0.0518 & 0.0394 & 0.0516 \\
0.5 & 0.1 & 0.2524 & 0.406 & 0.4068 & 0.4386 & 0.4392 & 0.215 & 0.3884 & 0.2164 & 0.401 \\
0.5 & 0.5 & 0.3272 & 0.5646 & 0.5652 & 0.5866 & 0.5832 & 0.2892 & 0.4976 & 0.2906 & 0.5556 \\
0.5 & 0.9 & 0.6458 & 0.9638 & 0.9628 & 0.9626 & 0.9624 & 0.6144 & 0.8562 & 0.61 & 0.9608 \\
1.0 & 0.1 & 0.5248 & 0.823 & 0.8216 & 0.8538 & 0.8356 & 0.471 & 0.8032 & 0.4802 & 0.8118 \\
1.0 & 0.5 & 0.633 & 0.9418 & 0.941 & 0.953 & 0.9546 & 0.5894 & 0.8988 & 0.5936 & 0.9374 \\
1.0 & 0.9 & 0.8764 & 0.9992 & 0.9992 & 0.9992 & 0.9992 & 0.8586 & 0.9914 & 0.8586 & 0.9992 \\
\hline
\end{tabular}


Table A.24. Heavy-Tailed Skewed Distribution with $n_{p}=20, n_{u x}=10, n_{u y}=10$. Empirical Size and Power at $\alpha=0.05$

\begin{tabular}{ccccccccccc}
\hline$\delta$ & $\rho$ & $T$ & $R_{z}$ & $R_{\text {perm }}$ & $R_{w, z}$ & $R_{w, \text { perm }}$ & $Z_{b}$ & $M$ & $t$ & $S$ \\
\hline 0 & 0.1 & 0.048 & 0.049 & 0.0486 & 0.0504 & 0.0514 & 0.0374 & 0.054 & 0.038 & 0.0482 \\
0 & 0.5 & 0.0494 & 0.048 & 0.0498 & 0.0494 & 0.05 & 0.0494 & 0.0524 & 0.0374 & 0.047 \\
0 & 0.9 & 0.052 & 0.0482 & 0.0492 & 0.0498 & 0.0506 & 0.0702 & 0.0482 & 0.037 & 0.0496 \\
0.5 & 0.1 & 0.2412 & 0.3878 & 0.3864 & 0.396 & 0.3956 & 0.1158 & 0.4052 & 0.1838 & 0.2806 \\
0.5 & 0.5 & 0.2944 & 0.493 & 0.4934 & 0.5 & 0.501 & 0.1906 & 0.4864 & 0.2522 & 0.3998 \\
0.5 & 0.9 & 0.5786 & 0.8698 & 0.8688 & 0.8712 & 0.8698 & 0.549 & 0.797 & 0.5656 & 0.8512 \\
1.0 & 0.1 & 0.5006 & 0.8116 & 0.8094 & 0.8174 & 0.8172 & 0.318 & 0.8308 & 0.4188 & 0.6376 \\
1.0 & 0.5 & 0.5824 & 0.911 & 0.9058 & 0.913 & 0.9108 & 0.4676 & 0.9008 & 0.5326 & 0.7934 \\
1.0 & 0.9 & 0.8328 & 0.9944 & 0.9936 & 0.9946 & 0.9936 & 0.8064 & 0.9858 & 0.8172 & 0.9886 \\
\hline
\end{tabular}

Table A.25. Heavy-Tailed Symmetric Distribution with $n_{p}=10, n_{u x}=0, n_{u y}=0$. Empirical Size and Power at $\alpha=0.05$

\begin{tabular}{ccccccccccc}
\hline$\delta$ & $\rho$ & $T$ & $R_{z}$ & $R_{\text {perm }}$ & $R_{w, z}$ & $R_{w, \text { perm }}$ & $Z_{b}$ & $M$ & $t$ & $S$ \\
\hline 0 & 0.1 & 0.0496 & 0.0536 & 0.0472 & 0.0536 & 0.0472 & 0.0416 & 0.0536 & 0.0416 & 0.0536 \\
0 & 0.5 & 0.0486 & 0.0538 & 0.0472 & 0.0538 & 0.0472 & 0.0412 & 0.0538 & 0.0412 & 0.0538 \\
0 & 0.9 & 0.0494 & 0.054 & 0.046 & 0.054 & 0.046 & 0.0398 & 0.054 & 0.0398 & 0.054 \\
0.5 & 0.1 & 0.1648 & 0.1826 & 0.1626 & 0.1826 & 0.1626 & 0.1446 & 0.1826 & 0.1446 & 0.1826 \\
0.5 & 0.5 & 0.2228 & 0.2374 & 0.2174 & 0.2374 & 0.2174 & 0.1954 & 0.2374 & 0.1954 & 0.2374 \\
0.5 & 0.9 & 0.5538 & 0.601 & 0.5612 & 0.601 & 0.5612 & 0.5136 & 0.601 & 0.5136 & 0.601 \\
1.0 & 0.1 & 0.37 & 0.414 & 0.3778 & 0.414 & 0.3778 & 0.3408 & 0.414 & 0.3408 & 0.414 \\
1.0 & 0.5 & 0.5016 & 0.5514 & 0.5104 & 0.5514 & 0.5104 & 0.4702 & 0.5514 & 0.4702 & 0.5514 \\
1.0 & 0.9 & 0.8558 & 0.9224 & 0.8994 & 0.9224 & 0.8994 & 0.8412 & 0.9224 & 0.8412 & 0.9224 \\
\hline
\end{tabular}

Table A.26. Heavy-Tailed Symmetric Distribution with $n_{p}=5, n_{u x}=2, n_{u y}=3$. Empirical Size and Power at $\alpha=0.05$

\begin{tabular}{ccccccccccc}
\hline$\delta$ & $\rho$ & $T$ & $R_{z}$ & $R_{\text {perm }}$ & $R_{w, z}$ & $R_{w, \text { perm }}$ & $Z_{b}$ & $M$ & $t$ & $S$ \\
\hline 0 & 0.1 & 0.061 & 0.045 & 0.0404 & 0.05 & 0.0488 & 0.0806 & 0.0612 & 0.0412 & 0.0672 \\
0 & 0.5 & 0.063 & 0.0472 & 0.0428 & 0.0542 & 0.052 & 0.085 & 0.064 & 0.0398 & 0.067 \\
0 & 0.9 & 0.0598 & 0.0466 & 0.0424 & 0.054 & 0.0504 & 0.0902 & 0.0624 & 0.0392 & 0.0672 \\
0.5 & 0.1 & 0.1688 & 0.143 & 0.1318 & 0.1612 & 0.1536 & 0.115 & 0.181 & 0.1108 & 0.1602 \\
0.5 & 0.5 & 0.193 & 0.1726 & 0.1616 & 0.1924 & 0.1852 & 0.15 & 0.205 & 0.1383 & 0.1944 \\
0.5 & 0.9 & 0.37 & 0.3402 & 0.3156 & 0.3536 & 0.3416 & 0.3614 & 0.343 & 0.339 & 0.4206 \\
1.0 & 0.1 & 0.3262 & 0.3084 & 0.2844 & 0.3346 & 0.318 & 0.2126 & 0.3588 & 0.2298 & 0.3044 \\
1.0 & 0.5 & 0.4026 & 0.3808 & 0.3576 & 0.4048 & 0.392 & 0.3048 & 0.42 & 0.3092 & 0.3888 \\
1.0 & 0.9 & 0.6992 & 0.6604 & 0.6306 & 0.6708 & 0.6494 & 0.7014 & 0.6082 & 0.6916 & 0.7572 \\
\hline
\end{tabular}


Table A.27. Heavy-Tailed Symmetric Distribution with $n_{p}=20, n_{u x}=0, n_{u y}=0$. Empirical Size and Power at $\alpha=0.05$

\begin{tabular}{ccccccccccc}
\hline$\delta$ & $\rho$ & $T$ & $R_{z}$ & $R_{\text {perm }}$ & $R_{w, z}$ & $R_{w, \text { perm }}$ & $Z_{b}$ & $M$ & $t$ & $S$ \\
\hline 0 & 0.1 & 0.051 & 0.0462 & 0.0486 & 0.0462 & 0.0486 & 0.0418 & 0.0462 & 0.0418 & 0.0462 \\
0 & 0.5 & 0.051 & 0.0464 & 0.05 & 0.0464 & 0.05 & 0.0418 & 0.0464 & 0.0418 & 0.0464 \\
0 & 0.9 & 0.0542 & 0.046 & 0.0494 & 0.046 & 0.0494 & 0.0406 & 0.046 & 0.0406 & 0.046 \\
0.5 & 0.1 & 0.2214 & 0.2668 & 0.2666 & 0.2668 & 0.2666 & 0.204 & 0.2668 & 0.204 & 0.26668 \\
0.5 & 0.5 & 0.3084 & 0.3706 & 0.3724 & 0.3706 & 0.3724 & 0.2864 & 0.3706 & 0.2864 & 0.3706 \\
0.5 & 0.9 & 0.687 & 0.8496 & 0.8484 & 0.8496 & 0.8484 & 0.662 & 0.8496 & 0.662 & 0.8496 \\
1.0 & 0.1 & 0.504 & 0.6228 & 0.6226 & 0.6228 & 0.6226 & 0.484 & 0.6228 & 0.484 & 0.6228 \\
1.0 & 0.5 & 0.6486 & 0.7958 & 0.793 & 0.7958 & 0.793 & 0.6296 & 0.7958 & 0.6296 & 0.7958 \\
1.0 & 0.9 & 0.92 & 0.9946 & 0.9944 & 0.9946 & 0.9944 & 0.9136 & 0.9946 & 0.9136 & 0.9946 \\
\hline
\end{tabular}

Table A.28. Heavy-Tailed Symmetric Distribution with $n_{p}=15, n_{u x}=2, n_{u y}=3$. Empirical Size and Power at $\alpha=0.05$

\begin{tabular}{ccccccccccc}
\hline$\delta$ & $\rho$ & $T$ & $R_{z}$ & $R_{\text {perm }}$ & $R_{w, z}$ & $R_{w, \text { perm }}$ & $Z_{b}$ & $M$ & $t$ & $S$ \\
\hline 0 & 0.1 & 0.0514 & 0.0458 & 0.0466 & 0.0498 & 0.0484 & 0.0936 & 0.053 & 0.0422 & 0.0536 \\
0 & 0.5 & 0.0504 & 0.0466 & 0.0472 & 0.0484 & 0.0504 & 0.0954 & 0.0518 & 0.042 & 0.053 \\
0 & 0.9 & 0.05 & 0.0462 & 0.046 & 0.0488 & 0.05 & 0.0934 & 0.0512 & 0.0396 & 0.052 \\
0.5 & 0.1 & 0.2164 & 0.237 & 0.2338 & 0.2538 & 0.248 & 0.1934 & 0.2408 & 0.185 & 0.2408 \\
0.5 & 0.5 & 0.2778 & 0.3192 & 0.3144 & 0.3334 & 0.332 & 0.2604 & 0.2918 & 0.248 & 0.3264 \\
0.5 & 0.9 & 0.6236 & 0.7426 & 0.7346 & 0.7448 & 0.7406 & 0.6172 & 0.573 & 0.6014 & 0.7524 \\
1.0 & 0.1 & 0.4726 & 0.546 & 0.5434 & 0.5874 & 0.5814 & 0.4222 & 0.5414 & 0.4172 & 0.538 \\
1.0 & 0.5 & 0.5964 & 0.6996 & 0.6956 & 0.727 & 0.7246 & 0.5634 & 0.6452 & 0.5576 & 0.6956 \\
1.0 & 0.9 & 0.8954 & 0.9786 & 0.9768 & 0.9784 & 0.9766 & 0.89 & 0.8814 & 0.888 & 0.9794 \\
\hline
\end{tabular}

Table A.29. Heavy-Tailed Symmetric Distribution with $n_{p}=10, n_{u x}=5, n_{u y}=5$. Empirical Size and Power at $\alpha=0.05$

\begin{tabular}{ccccccccccc}
\hline$\delta$ & $\rho$ & $T$ & $R_{z}$ & $R_{\text {perm }}$ & $R_{w, z}$ & $R_{w, \text { perm }}$ & $Z_{b}$ & $M$ & $t$ & $S$ \\
\hline 0 & 0.1 & 0.056 & 0.0564 & 0.0476 & 0.0528 & 0.0538 & 0.0604 & 0.0502 & 0.0416 & 0.0542 \\
0 & 0.5 & 0.0542 & 0.0562 & 0.0474 & 0.0512 & 0.0532 & 0.069 & 0.05 & 0.0416 & 0.0542 \\
0 & 0.9 & 0.0512 & 0.0542 & 0.0466 & 0.0514 & 0.0522 & 0.085 & 0.05 & 0.0398 & 0.0534 \\
0.5 & 0.1 & 0.2042 & 0.2502 & 0.2206 & 0.2386 & 0.2366 & 0.114 & 0.237 & 0.1498 & 0.1868 \\
0.5 & 0.5 & 0.2482 & 0.3108 & 0.2784 & 0.2958 & 0.2924 & 0.1714 & 0.273 & 0.1988 & 0.247 \\
0.5 & 0.9 & 0.5236 & 0.6226 & 0.5842 & 0.5976 & 0.596 & 0.5022 & 0.5032 & 0.5068 & 0.5962 \\
1.0 & 0.1 & 0.4492 & 0.5636 & 0.5188 & 0.5482 & 0.5412 & 0.272 & 0.5478 & 0.3448 & 0.4142 \\
1.0 & 0.5 & 0.5344 & 0.6716 & 0.639 & 0.6594 & 0.6582 & 0.4134 & 0.636 & 0.465 & 0.541 \\
1.0 & 0.9 & 0.8448 & 0.9348 & 0.9194 & 0.9246 & 0.9236 & 0.829 & 0.8522 & 0.8342 & 0.9188 \\
\hline
\end{tabular}


Table A.30. Heavy-Tailed Symmetric Distribution with $n_{p}=5, n_{u x}=7, n_{u y}=8$. Empirical Size and Power at $\alpha=0.05$

\begin{tabular}{ccccccccccc}
\hline$\delta$ & $\rho$ & $T$ & $R_{z}$ & $R_{\text {perm }}$ & $R_{w, z}$ & $R_{w, \text { perm }}$ & $Z_{b}$ & $M$ & $t$ & $S$ \\
\hline 0 & 0.1 & 0.0658 & 0.0596 & 0.0494 & 0.0498 & 0.0504 & 0.029 & 0.0532 & 0.0362 & 0.0612 \\
0 & 0.5 & 0.0644 & 0.0586 & 0.0482 & 0.0536 & 0.0538 & 0.0418 & 0.0554 & 0.037 & 0.0616 \\
0 & 0.9 & 0.0624 & 0.061 & 0.051 & 0.0512 & 0.0536 & 0.0652 & 0.0546 & 0.0382 & 0.0614 \\
0.5 & 0.1 & 0.2012 & 0.2346 & 0.206 & 0.2154 & 0.2142 & 0.0452 & 0.225 & 0.107 & 0.1572 \\
0.5 & 0.5 & 0.2318 & 0.2548 & 0.2254 & 0.2504 & 0.249 & 0.0758 & 0.2576 & 0.1368 & 0.1904 \\
0.5 & 0.9 & 0.3828 & 0.3318 & 0.2934 & 0.3992 & 0.3984 & 0.284 & 0.406 & 0.3428 & 0.4302 \\
1.0 & 0.1 & 0.4194 & 0.527 & 0.4806 & 0.5068 & 0.5022 & 0.0846 & 0.5164 & 0.2204 & 0.2992 \\
1.0 & 0.5 & 0.4726 & 0.5558 & 0.5134 & 0.5658 & 0.5664 & 0.1516 & 0.5756 & 0.3102 & 0.392 \\
1.0 & 0.9 & 0.709 & 0.6526 & 0.6106 & 0.7684 & 0.769 & 0.6188 & 0.7712 & 0.6974 & 0.7636 \\
\hline
\end{tabular}

Table A.31. Heavy-Tailed Symmetric Distribution with $n_{p}=40, n_{u x}=0, n_{u y}=0$. Empirical Size and Power at $\alpha=0.05$

\begin{tabular}{ccccccccccc}
\hline$\delta$ & $\rho$ & $T$ & $R_{z}$ & $R_{\text {perm }}$ & $R_{w, z}$ & $R_{w, \text { perm }}$ & $Z_{b}$ & $M$ & $t$ & $S$ \\
\hline 0 & 0.1 & 0.0492 & 0.0464 & 0.0496 & 0.0464 & 0.0496 & 0.0454 & 0.0464 & 0.0454 & 0.0464 \\
0 & 0.5 & 0.0502 & 0.047 & 0.049 & 0.047 & 0.049 & 0.0442 & 0.047 & 0.0442 & 0.047 \\
0 & 0.9 & 0.0512 & 0.048 & 0.0492 & 0.048 & 0.0492 & 0.0418 & 0.048 & 0.0418 & 0.048 \\
0.5 & 0.1 & 0.324 & 0.4464 & 0.4432 & 0.4464 & 0.4432 & 0.3064 & 0.4464 & 0.3064 & 0.4464 \\
0.5 & 0.5 & 0.4376 & 0.6024 & 0.6016 & 0.6024 & 0.6016 & 0.4188 & 0.6024 & 0.4188 & 0.6024 \\
0.5 & 0.9 & 0.8126 & 0.9892 & 0.9872 & 0.9892 & 0.9872 & 0.7964 & 0.9892 & 0.7964 & 0.9892 \\
1.0 & 0.1 & 0.6802 & 0.8852 & 0.884 & 0.8852 & 0.884 & 0.664 & 0.8852 & 0.664 & 0.8852 \\
1.0 & 0.5 & 0.8056 & 0.9772 & 0.9762 & 0.9772 & 0.9762 & 0.791 & 0.9779 & 0.711 & 0.9772 \\
1.0 & 0.9 & 0.9606 & 1 & 1 & 1 & 1 & 0.9554 & 1 & 0.9554 & 1 \\
\hline
\end{tabular}

Table A.32. Heavy-Tailed Symmetric Distribution with $n_{p}=20, n_{u x}=10, n_{u y}=10$. Empirical Size and Power at $\alpha=0.05$

\begin{tabular}{ccccccccccc}
\hline$\delta$ & $\rho$ & $T$ & $R_{z}$ & $R_{\text {perm }}$ & $R_{w, z}$ & $R_{w, \text { perm }}$ & $Z_{b}$ & $M$ & $t$ & $S$ \\
\hline 0 & 0.1 & 0.0534 & 0.0498 & 0.0504 & 0.0516 & 0.0538 & 0.0458 & 0.0528 & 0.0436 & 0.0472 \\
0 & 0.5 & 0.0528 & 0.0486 & 0.0492 & 0.0498 & 0.0512 & 0.0594 & 0.0526 & 0.0438 & 0.0464 \\
0 & 0.9 & 0.051 & 0.0478 & 0.0494 & 0.0494 & 0.0508 & 0.0814 & 0.0512 & 0.0434 & 0.0454 \\
0.5 & 0.1 & 0.2848 & 0.3678 & 0.3674 & 0.378 & 0.3784 & 0.1452 & 0.3808 & 0.2186 & 0.278 \\
0.5 & 0.5 & 0.3504 & 0.4716 & 0.4708 & 0.4774 & 0.477 & 0.2388 & 0.4564 & 0.2926 & 0.3806 \\
0.5 & 0.9 & 0.6998 & 0.8788 & 0.8744 & 0.879 & 0.8762 & 0.661 & 0.7922 & 0.6762 & 0.8528 \\
1.0 & 0.1 & 0.6 & 0.8068 & 0.8054 & 0.813 & 0.8114 & 0.3916 & 0.82 & 0.5044 & 0.6384 \\
1.0 & 0.5 & 0.7062 & 0.9092 & 0.9082 & 0.9122 & 0.9122 & 0.5792 & 0.8998 & 0.6368 & 0.8004 \\
1.0 & 0.9 & 0.9246 & 0.9976 & 0.9974 & 0.9974 & 0.9974 & 0.9086 & 0.9904 & 0.914 & 0.9964 \\
\hline
\end{tabular}


Table A.33. Heavy-Tailed Symmetric Distribution with $n_{p}=8, n_{u x}=16, n_{u y}=16$. Empirical Size and Power at $\alpha=0.05$

\begin{tabular}{ccccccccccc}
\hline$\delta$ & $\rho$ & $T$ & $R_{z}$ & $R_{\text {perm }}$ & $R_{w, z}$ & $R_{w, \text { perm }}$ & $Z_{b}$ & $M$ & $t$ & $S$ \\
\hline 0 & 0.1 & 0.0602 & 0.0508 & 0.0516 & 0.0532 & 0.0532 & 0.015 & 0.052 & 0.035 & 0.0496 \\
0 & 0.5 & 0.06 & 0.0526 & 0.0538 & 0.053 & 0.0522 & 0.027 & 0.0518 & 0.037 & 0.0496 \\
0 & 0.9 & 0.0562 & 0.0502 & 0.0502 & 0.0518 & 0.0532 & 0.0554 & 0.0518 & 0.0362 & 0.0492 \\
0.5 & 0.1 & 0.2516 & 0.3242 & 0.32 & 0.3446 & 0.341 & 0.0246 & 0.342 & 0.1334 & 0.1722 \\
0.5 & 0.5 & 0.2792 & 0.3376 & 0.337 & 0.3862 & 0.3878 & 0.0584 & 0.386 & 0.1768 & 0.2212 \\
0.5 & 0.9 & 0.4892 & 0.4136 & 0.4088 & 0.6078 & 0.6034 & 0.366 & 0.615 & 0.4892 & 0.5136 \\
1.0 & 0.1 & 0.5508 & 0.7456 & 0.7424 & 0.7612 & 0.7646 & 0.0626 & 0.7588 & 0.3016 & 0.3496 \\
1.0 & 0.5 & 0.6052 & 0.773 & 0.767 & 0.8302 & 0.8302 & 0.1702 & 0.8294 & 0.4226 & 0.4754 \\
1.0 & 0.9 & 0.8252 & 0.8358 & 0.8296 & 0.954 & 0.9518 & 0.7356 & 0.9552 & 0.8028 & 0.8238 \\
\hline
\end{tabular}




\section{APPENDIX B}

R CODE

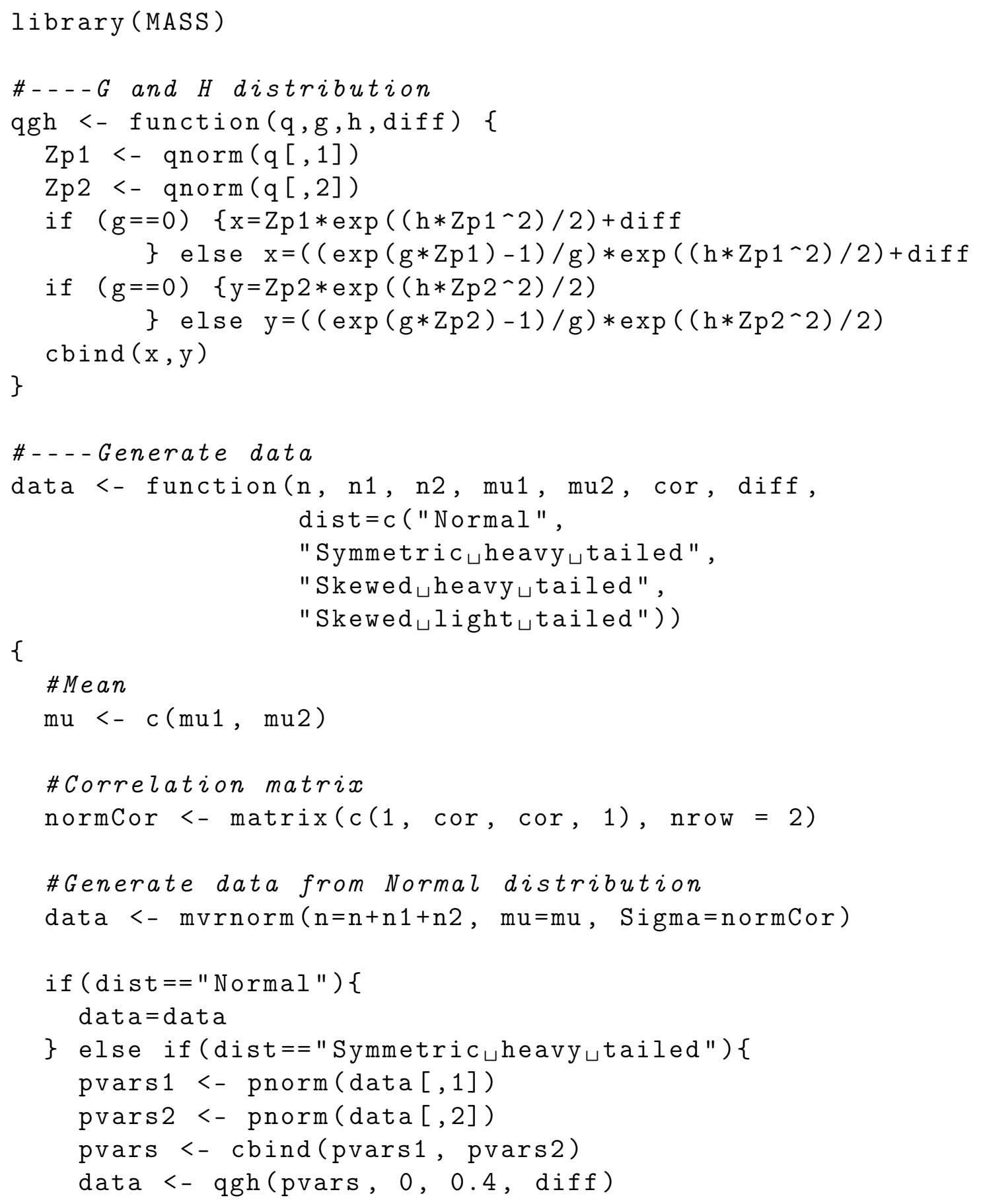




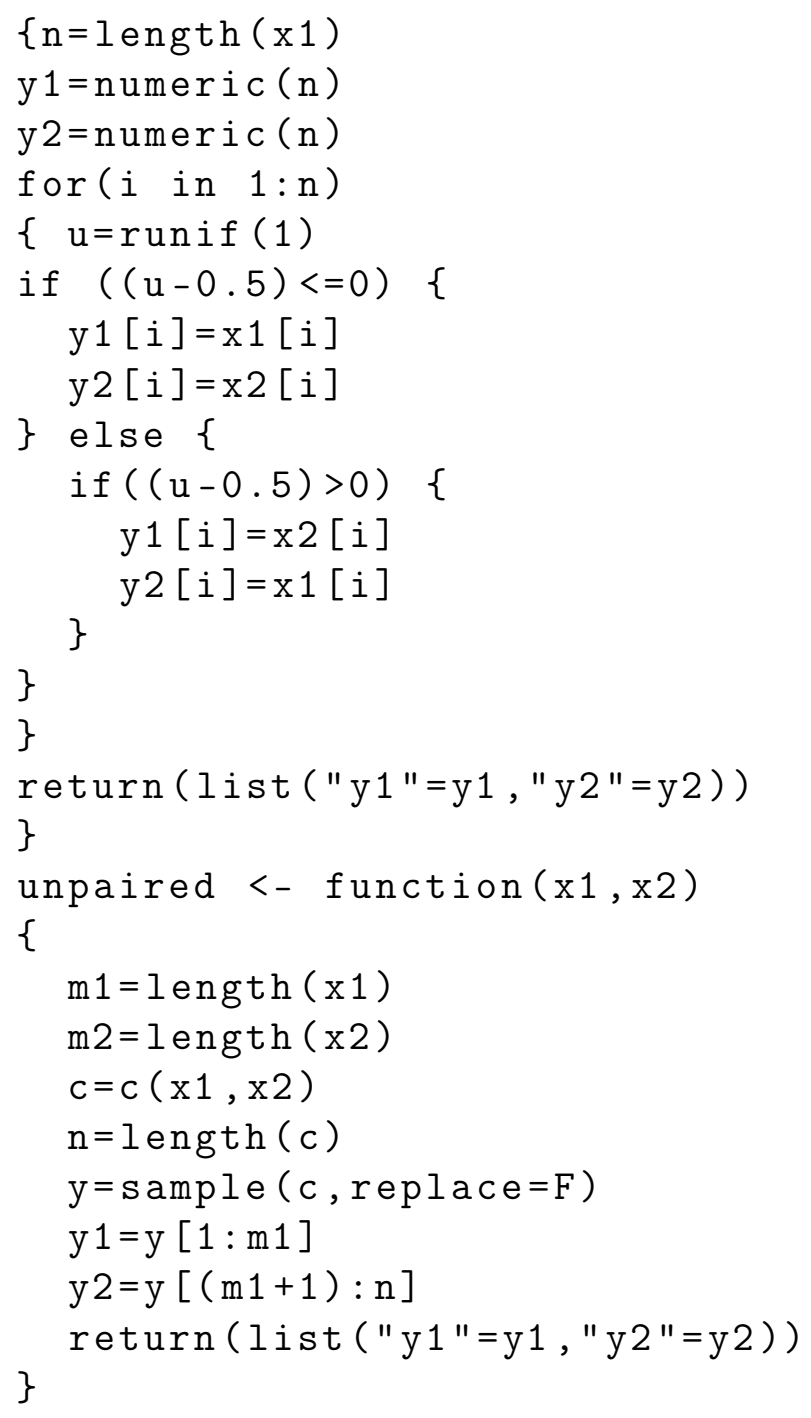




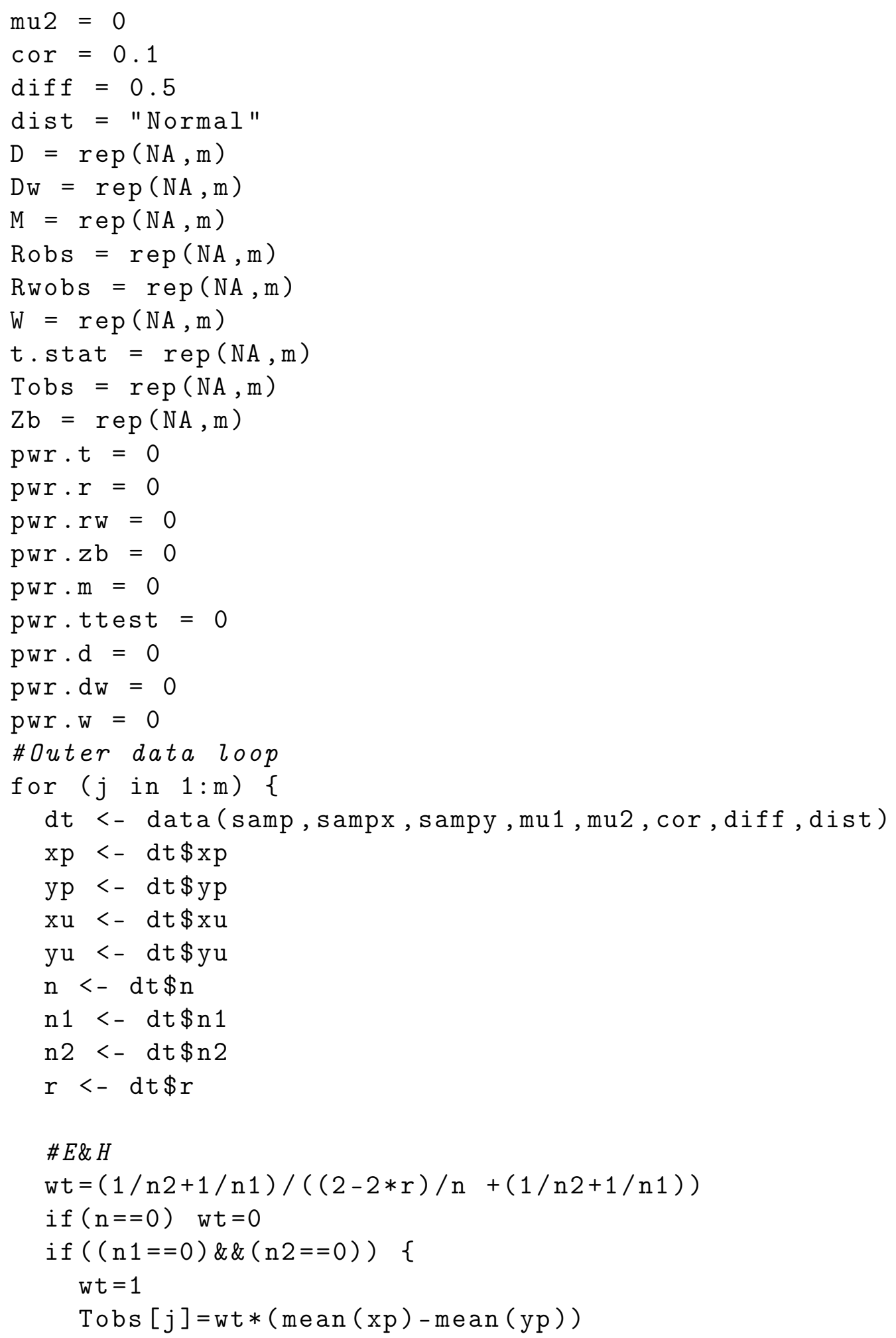




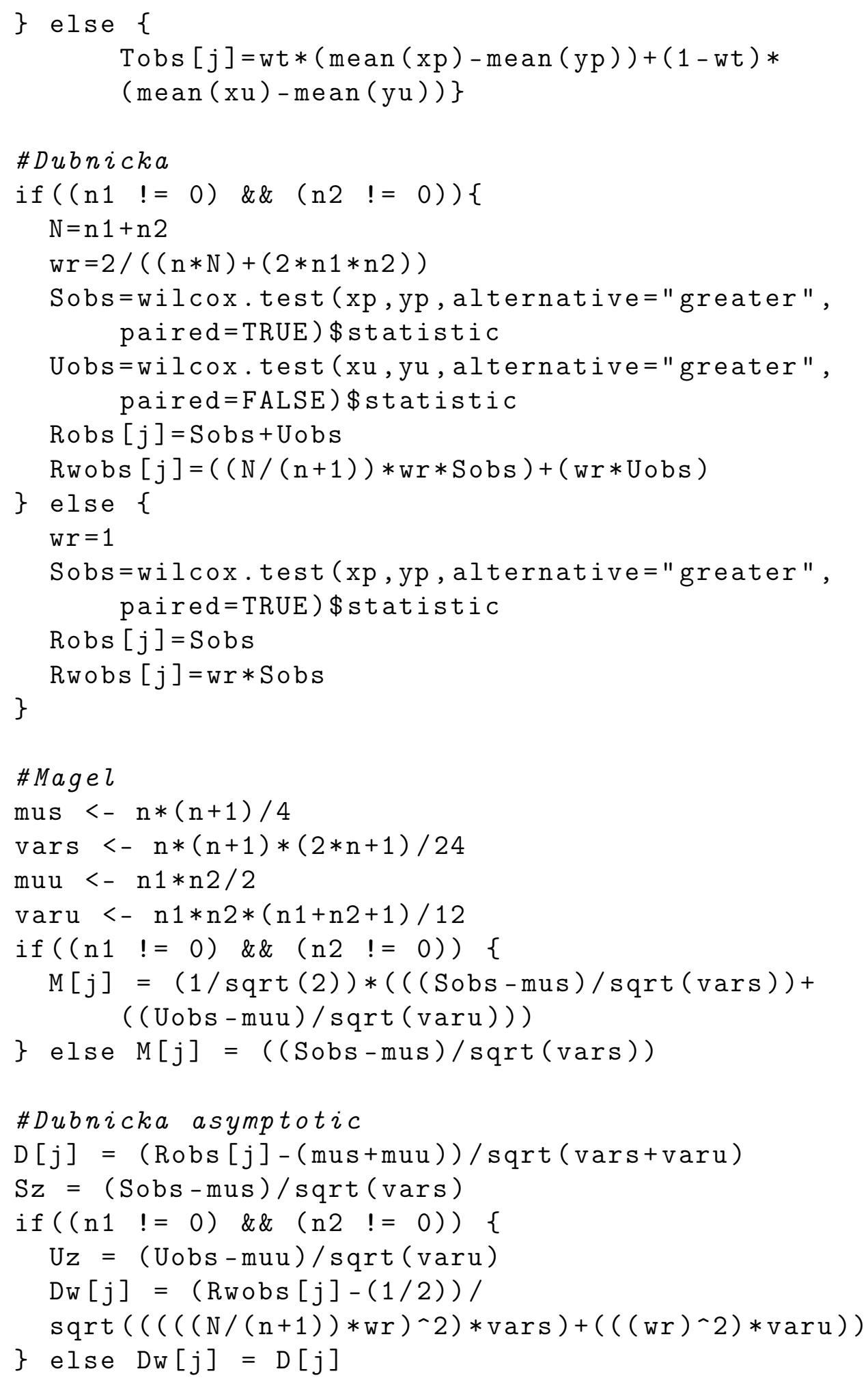




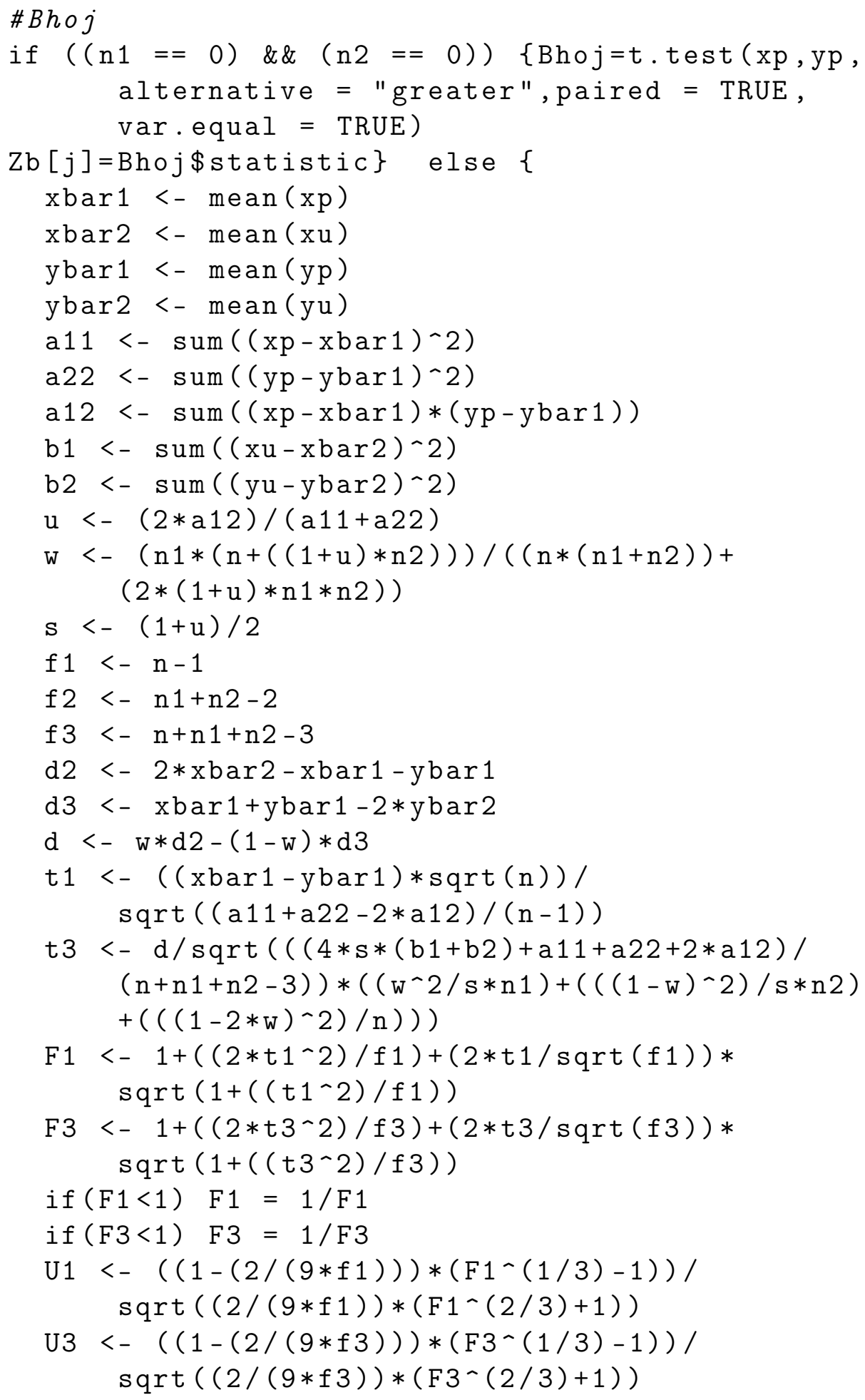




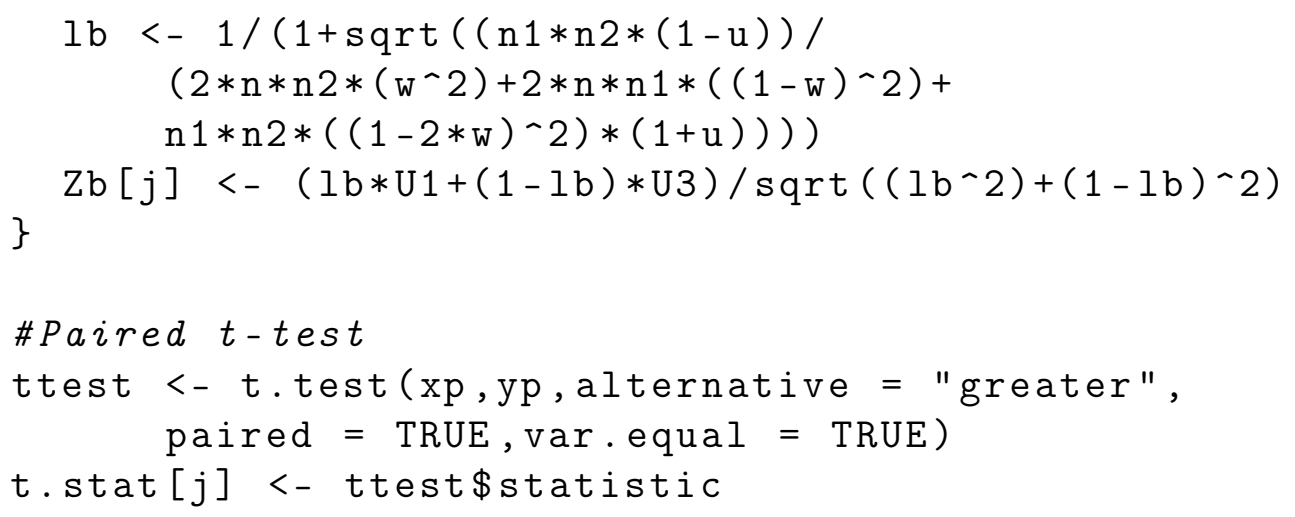




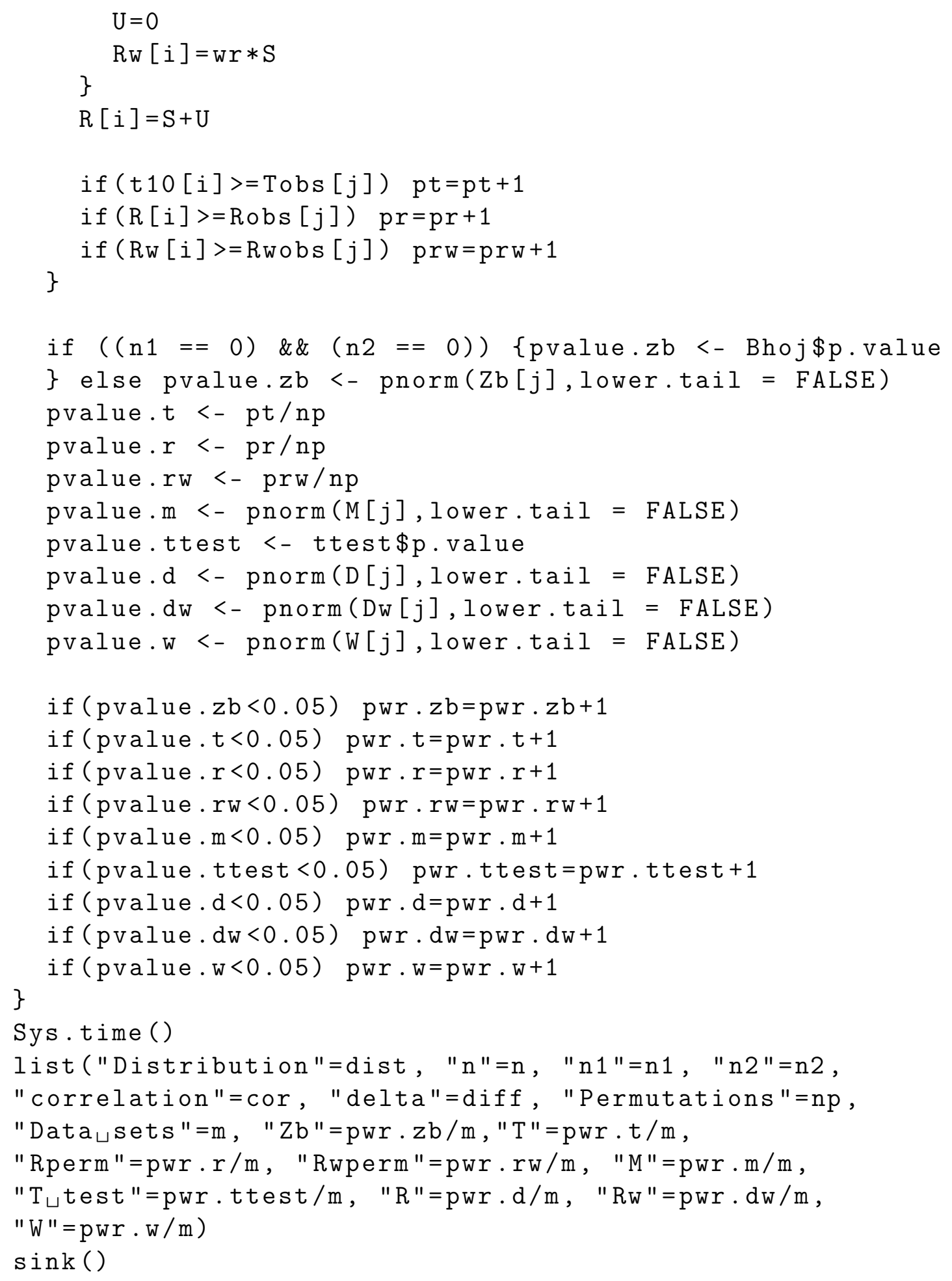




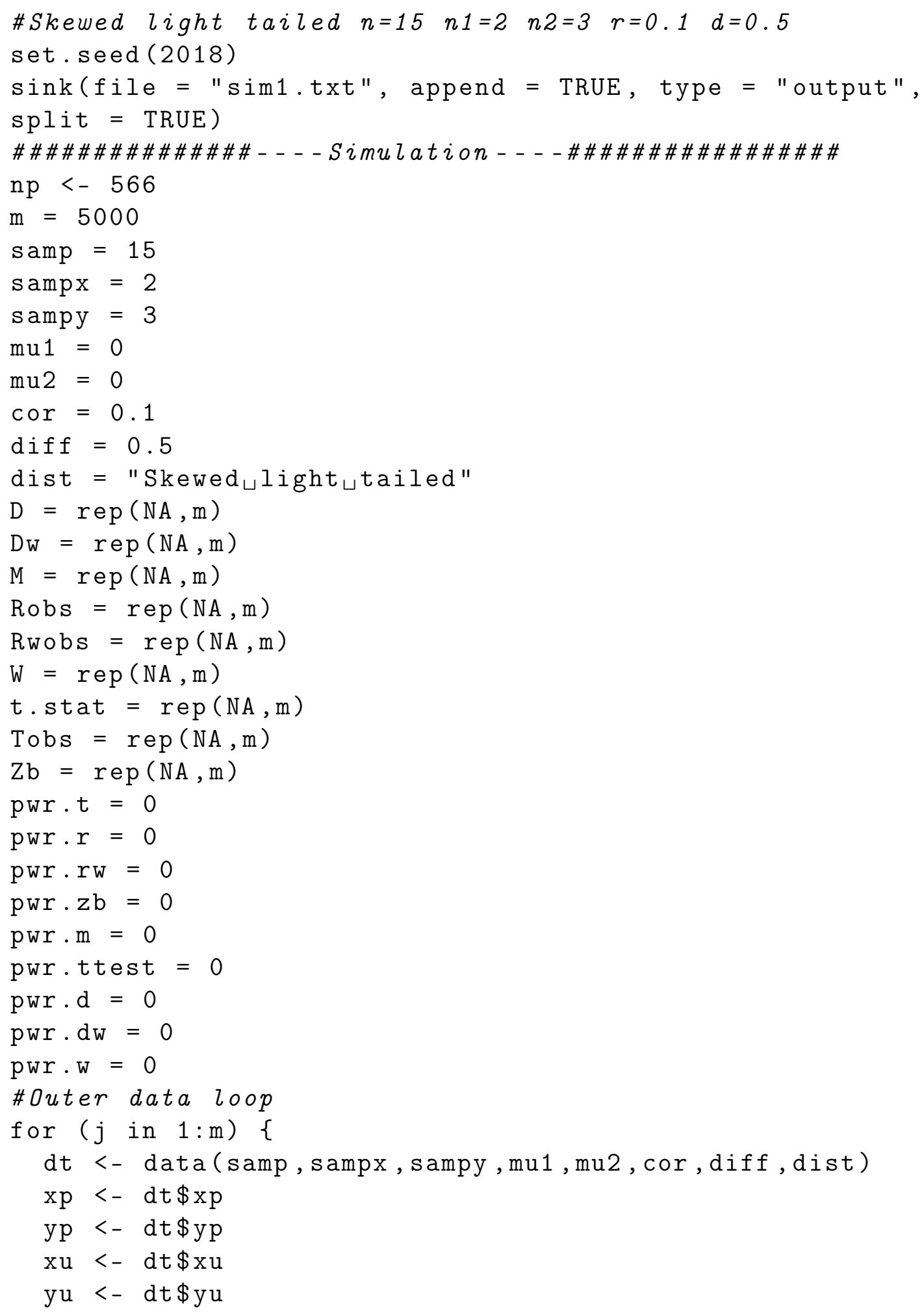




$$
\begin{aligned}
& \mathrm{n}<-\operatorname{dt} \$ \mathrm{n} \\
& \mathrm{n} 1<-\operatorname{dt} \$ \mathrm{n} 1 \\
& \mathrm{n} 2<-\operatorname{dt} \$ \mathrm{n} 2 \\
& \mathrm{r}<-\operatorname{dt} \$ \mathrm{r}
\end{aligned}
$$

\section{$\# E \& H$}

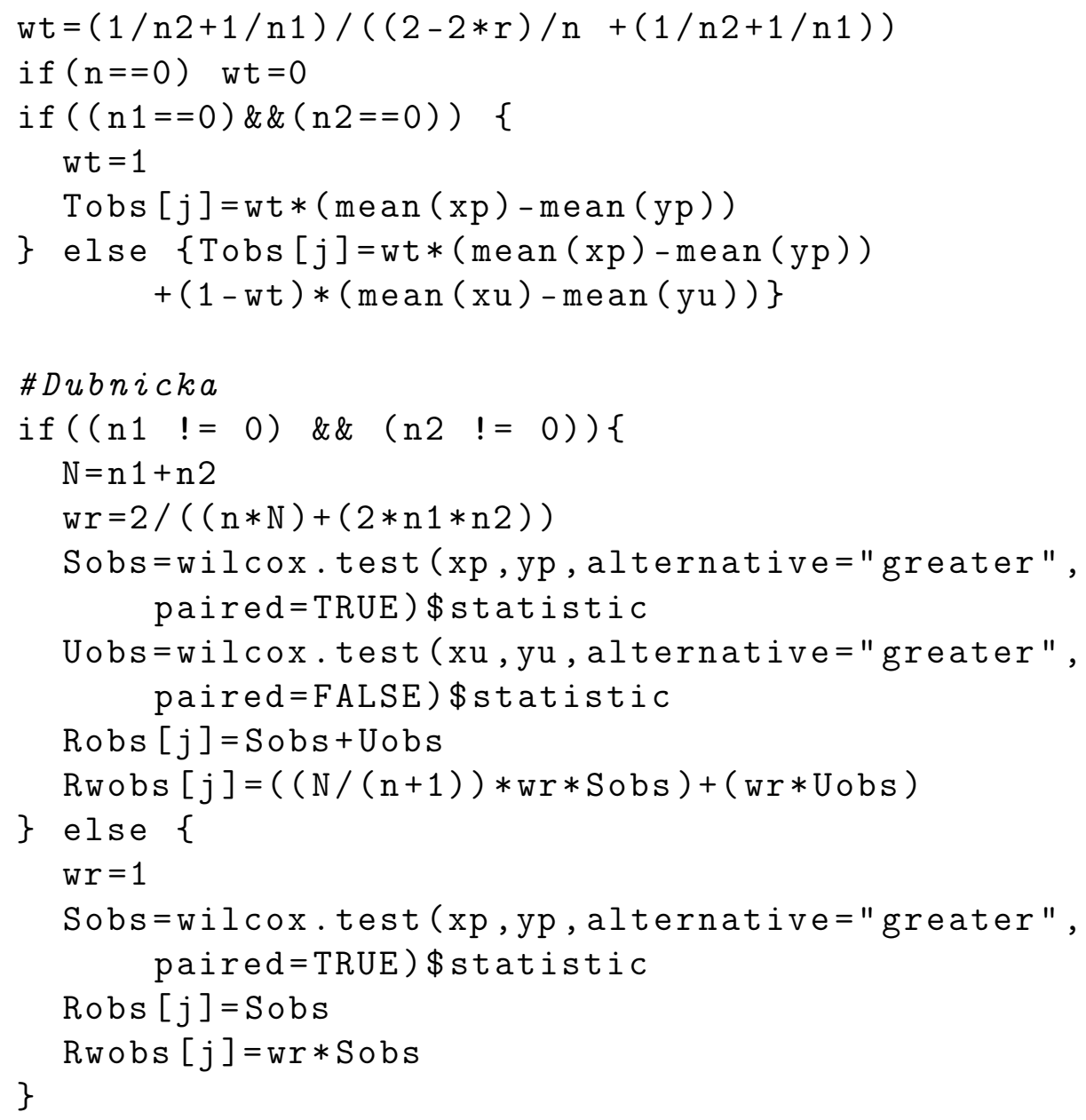


\} else $M[j]=(($ Sobs-mus $) / \operatorname{sqrt}(\operatorname{vars}))$

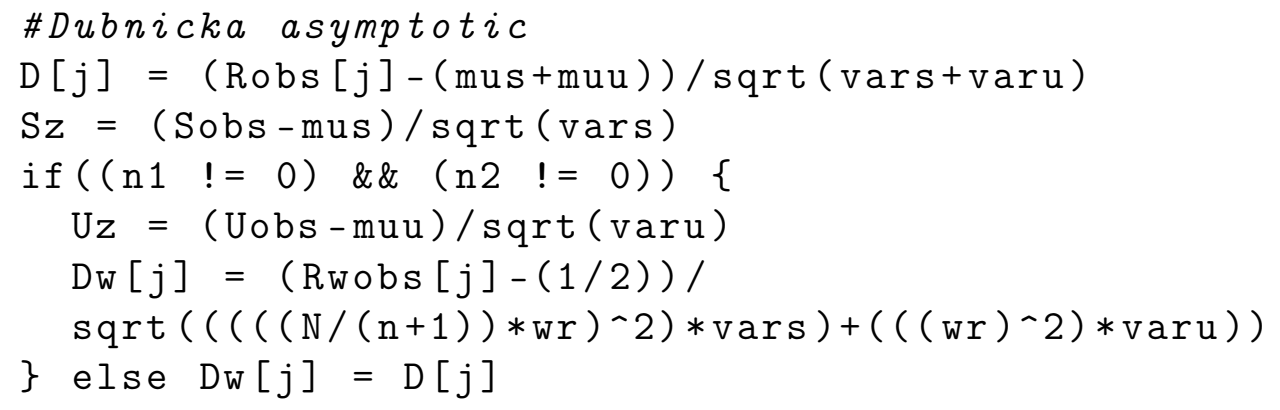

\section{$\# B h \circ j$}

if $((n 1==0) \& \&(n 2==0)) \quad\{B h \circ j=t$.test (xp, yp, alternative = "greater", paired = TRUE, var. equal = TRUE)

$\mathrm{Zb}[\mathrm{j}]=$ Bhoj\$statistic $\}$ else \{ xbar $1<-\operatorname{mean}(\mathrm{xp})$ $\mathrm{xbar} 2<-\operatorname{mean}(\mathrm{xu})$

ybar $1<-\operatorname{mean}(y p)$ ybar $2<-\operatorname{mean}(y u)$

a11<- $\operatorname{sum}((x p-x \operatorname{bar} 1)-2)$

a22<- $\operatorname{sum}((y p-y b a r 1)-2)$

a $12<-\operatorname{sum}((x p-x b a r 1) *(y p-y b a r 1))$

b1 $<-\operatorname{sum}((x u-x b \operatorname{ar} 2)-2)$

b2 <- $\operatorname{sum}((y u-y b a r 2)-2)$

$\mathrm{u}<-(2 * a 12) /(\mathrm{a} 11+\mathrm{a} 22)$

$\mathrm{w}<-(\mathrm{n} 1 *(\mathrm{n}+((1+\mathrm{u}) * \mathrm{n} 2))) /$ $((\mathrm{n} *(\mathrm{n} 1+\mathrm{n} 2))+(2 *(1+\mathrm{u}) * \mathrm{n} 1 * \mathrm{n} 2))$

$\mathrm{s}<-(1+u) / 2$

$\mathrm{f} 1<-\mathrm{n}-1$

$\mathrm{f} 2<-\mathrm{n} 1+\mathrm{n} 2-2$

f $3<-n+n 1+n 2-3$

d2 $<-2 * x b a r 2-x b a r 1-$ ybar 1

d3 $<-$ xbar $1+$ ybar $1-2 *$ ybar 2

$\mathrm{d}<-\mathrm{w} * \mathrm{~d} 2-(1-\mathrm{w}) * \mathrm{~d} 3$

t1 $<-((\operatorname{xbar} 1-\operatorname{ybar} 1) * \operatorname{sqrt}(\mathrm{n})) /$ $\operatorname{sqrt}((a 11+a 22-2 * a 12) /(n-1))$

t3 <- d/sqrt $(((4 * \mathrm{~s} *(\mathrm{~b} 1+\mathrm{b} 2)+\mathrm{a} 11+\mathrm{a} 22+2 * \mathrm{a} 12) /$ $(\mathrm{n}+\mathrm{n} 1+\mathrm{n} 2-3)) *((\mathrm{w} \wedge 2 / \mathrm{s} * \mathrm{n} 1)+(((1-\mathrm{w}) \wedge 2) / \mathrm{s} * \mathrm{n} 2)$ $+(((1-2 * \mathrm{w}) \wedge 2) / \mathrm{n})))$ 


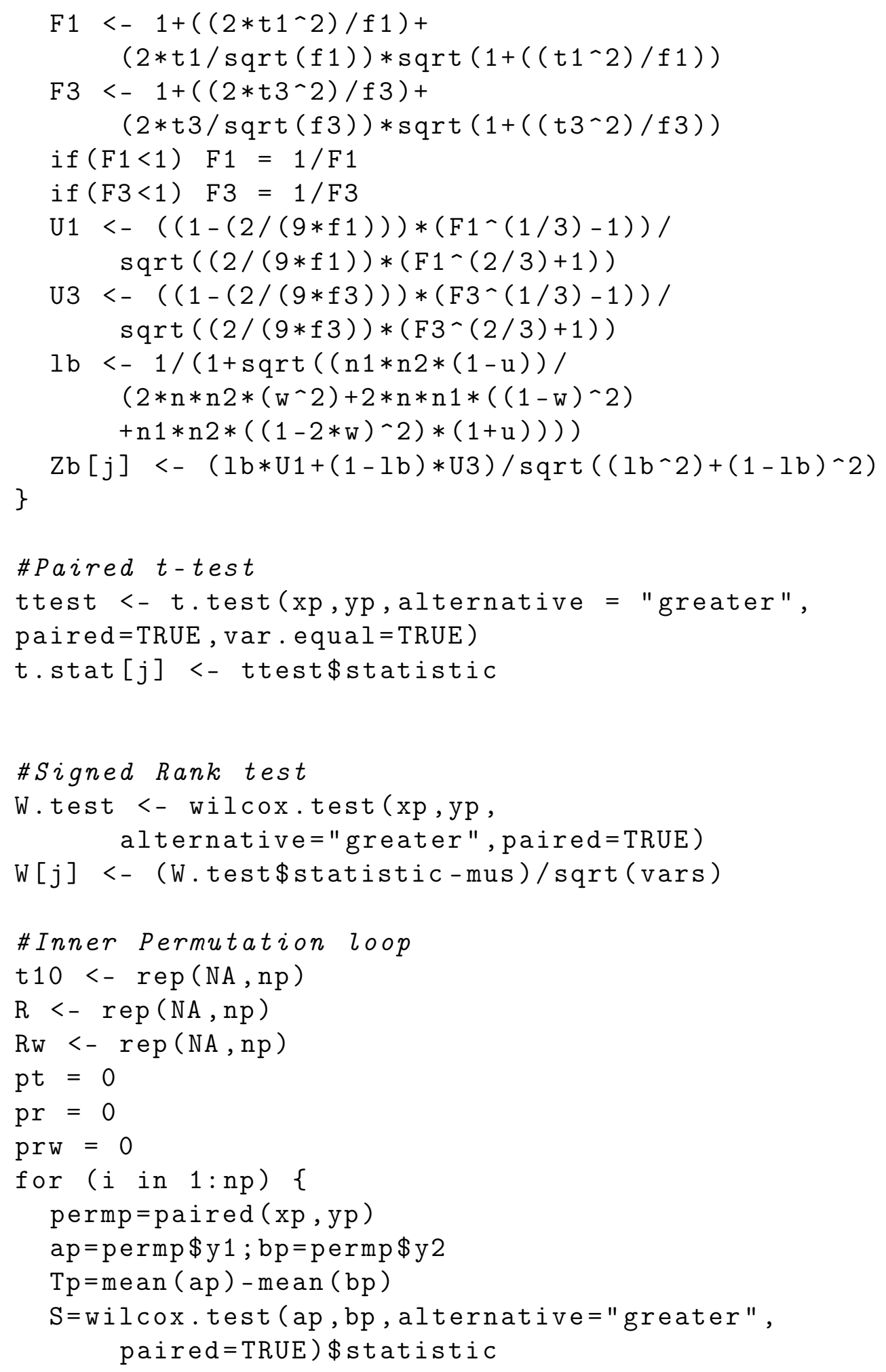




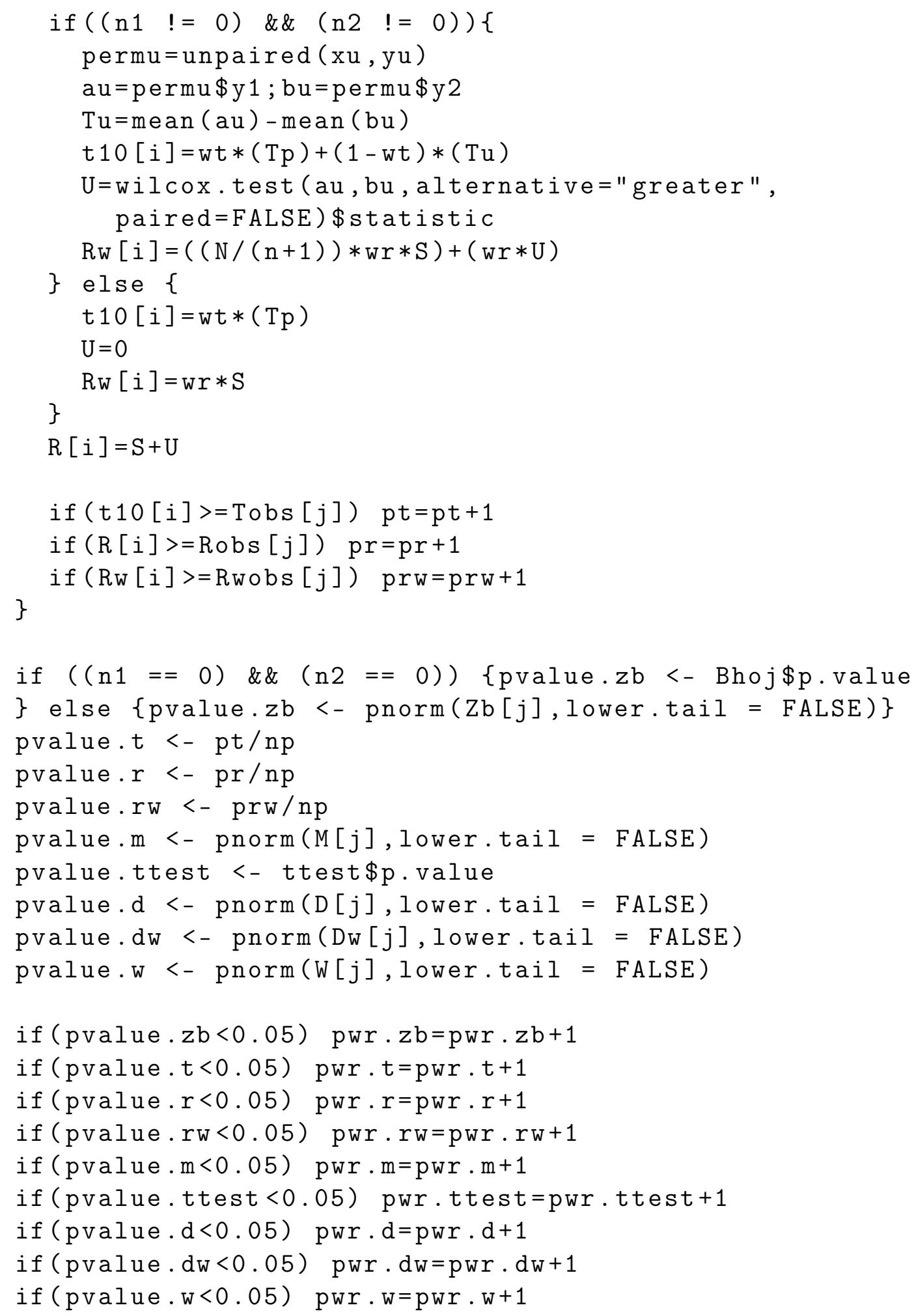


\}

Sys.time()

list ("Distribution"=dist, "n"=n, "n1"=n1, "n2"=n2,

"correlation"=cor, "delta"=diff, "Permutations"=np,

"Data sets"=m, "Zb"=pwr.zb/m, "T"=pwr.t/m,

"Rperm"=pwr.r/m, "Rwperm"=pwr.rw/m, "M"=pwr.m/m,

"T与test"=pwr.ttest/m, "R"=pwr.d/m, "Rw"=pwr.dw/m, "W" $=$ pwr. $\mathrm{W} / \mathrm{m}$ )

sink () 